\title{
Malaria Transmission Model with Transmission-Blocking Drugs and a Time Delay
}

\author{
Huike Wu and Zhixing Hu \\ School of Mathematics and Physics, University of Science and Technology Beijing, Beijing 100083, China \\ Correspondence should be addressed to Zhixing Hu; huzhixing@ustb.edu.cn
}

Received 17 June 2021; Accepted 25 August 2021; Published 11 September 2021

Academic Editor: Tongqian Zhang

Copyright ( 2021 Huike Wu and Zhixing Hu. This is an open access article distributed under the Creative Commons Attribution License, which permits unrestricted use, distribution, and reproduction in any medium, provided the original work is properly cited.

\begin{abstract}
A class of transmission-blocking drugs (TBDs) that block the transmission of parasites between humans and mosquitoes has recently been shown to be effective in controlling malaria transmission. In this paper, we develop a time-delay differential equation model for malaria using TBDs intervention, in which the human population consists of a treated class and a successfully treated class. In classifying the positive equilibria, the control reproduction number $R_{T}$ was obtained and the forward and backward branching cases were explored. Then, by constructing a Lyapunov function, the disease-free equilibrium is globally asymptotically stable under certain conditions. In addition, when $R_{T}>1$, the model exhibits Hopf bifurcation, the positive equilibrium becomes unstable from stable, and the model exhibits a periodic solution due to the change of time delay. On the other hand, it is concluded that the use of TBDs has a positive effect on disease control when the treatment rate and the efficacy of TBDs meet certain conditions. Finally, numerical simulation was used to observe the effect of treatment rate and the efficacy of TBDs on $R_{T}$, and it was found that the increase in the efficacy of TBDs had a more pronounced effect on disease control compared to treatment rate.
\end{abstract}

\section{Introduction}

Malaria is an insect-borne disease caused by Plasmodium infection through the bite of Anopheles mosquitoes or by importing the blood of a person with Plasmodium and is a major infectious disease that seriously endangers people's health and life safety. The first symptoms of malaria usually appear 10-15 days after people are bitten by mosquitoes that have been infected with the virus. The disease mainly manifests as periodic regular attacks with generalized chills, fever, and excessive sweating, and after many longterm attacks, it can cause anemia and splenomegaly. Plasmodium falciparum can lead to the most serious diseases (see [1]). WHO once ranked malaria as one of the top three global public health problems, along with AIDS and Tuberculosis. Data from 1980 to 2010 show that malaria infection rates have declined, but mortality remains a concern. To date, malaria remains a serious global epidemic, with about $40 \%$ of the world's population living in malaria-endemic regions, $90 \%$ of which are on the African continent. More than 2 million people die from malaria each year, especially children under five years of age, pregnant women, and those suffering from other diseases (see [2]). The tropics and subtropics are the most malaria-prone regions, forming a "malaria belt" around the equator that includes Latin America, sub-Saharan Africa, South Asia, and Southeast Asia. WHO data show that, in 2017, there were an estimated 219 million cases of malaria in 87 countries, and in 2019, there were an estimated 229 million malaria cases worldwide, with an estimated 409,000 malaria deaths.

Early diagnosis and treatment of malaria can reduce disease and avert deaths, and since 2000, the expansion of vector control interventions has led to progress in malaria control, particularly in sub-Saharan Africa (see [3]). The best available treatments, particularly for falciparum malaria, are artemisinin-based combination therapies. However, these gains are jeopardized by the emergence of 
resistance to antimalarial drugs in Anopheles mosquitoes. Furthermore, some studies have shown an increase in resistance to artemisinin combination therapies paired with drugs (see $[4,5]$ ). Rather than relying on vector control to prevent mosquito bites, the transmission of gametocytes (the intraerythrocytic sexual stages) to the mosquito is potentially more amenable to direct intervention because it is easily targetable within the human blood compartment (see [6]). Most antimalarial drugs are inactive against the sexual stage of $P$. falciparum, and for effective long-term malaria control, measures are needed to stop the transmission of $P$. falciparum between humans and mosquitoes. Several studies have shown that a potential approach to directly block parasite transmission is to target Plasmodium using transmission-blocking interventions (TBIs), which can be broadly categorized as transmission-blocking drugs (TBDs) or transmissionblocking vaccines (TBVs) that target parasitic stages (see [7]). These drugs can be divided into two categories, one targeting the parasitic stage exposed only in mosquitoes and the other targeting the parasitic stage exposed only in humans. For TBDs that target parasites in humans, drugs can kill the asexual phase of the parasite, stopping/reducing development to gametes, drugs target immature and mature gametes in humans directly, or drugs provide chemotherapeutic prophylaxis by direct action on Sporozoa, thus stopping infection. From these aspects, there are many scholars working on transmission-blocking drugs that can block the different stages of the parasite within the host. Antimalarial drugs with transmissionblocking activity have been prioritized, and several are in various stages of clinical development (see [8]).

Exploring the dynamics of infectious diseases through mathematical modeling can be used to guide disease control. A number of mathematicians have explored the dynamics of malaria transmission between humans and mosquitoes by modeling differential equations. Following the studies of Ross (see [9]) and Macdonald (see [10]), a series of differential equation models for malaria have been proposed one after another from different perspectives (see [11-16]). In recent years, Ngwa proposed a deterministic differential equation model for malaria epidemics (see [17]), in which human and mosquito populations have SEIR and SEI structures, respectively, and most subsequent studies have followed this structure. Chitnis proposed a mathematical model for the transmission of malaria in populations and mosquitoes with ordinary differential equations (see [18]), and numerical simulations showed that, for larger disease lethality, subcritical (backward) bifurcation may occur when the basic reproduction number is equal to 1 . In 2008, Chitnis identified important parameters of malaria transmission by sensitivity analysis of the mathematical model (see [19]). Ruan considered the incubation period of the parasite in humans and mosquitoes and proposed a delayed Ross-Macdonald model (see [20]), which demonstrated that the basic reproduction number is a decreasing function of two time delays and that the prevalence of infection can be reduced by extending the incubation period in humans or mosquitoes. In the context of some progress in new antimalarial drug research, a regional deterministic model assessed the effectiveness of a malaria transmission-blocking vaccine targeting the parasitic stage of mosquitoes (see [21]). In addition, a mathematical model assessed the role of gametocytes (the infectious stage of the malaria parasite) in the dynamics of malaria transmission (see [22]), which was extended to include some hypothetical therapeutic features of an imperfect vaccine.

Mathematical models of drug in-host kinetics are often used to guide drug development, and these models often focus on assessing efficacy and response duration to guide patient treatment (see [23]). Based on this motivation, in this paper, we refer to the modeling ideas from the literature (see [24]) and add TBDs targeting parasite blockade in humans to the malaria model, assuming that a fraction of human infected patients are treated with TBDs and that a fraction of patients successfully block parasite transmission to mosquitoes after treatment with TBDs. In addition, usually, people develop the first symptoms of malaria after being infected with the virus for a period of time (see $[25,26])$. Being undetected during the incubation period leads to an increased risk of malaria infection in humans by not treating the parasite promptly or using TBDs to block transmission to mosquitoes in a timely manner. Based on these two considerations, this paper develops a differential equation model considering TBDs and a time delay and is dedicated to analyzing the effect of the incubation period of Plasmodium in humans on the stability of the model's equilibrium, as well as the effect of treatment with TBDs on malaria control in humans, and to assessing the range of treatment rate and the efficacy of TBDs that can contribute most to malaria control under certain conditions.

\section{Model Description}

In this model, the total human population $N_{h}$ is divided into susceptible individuals $S_{h}$, infected individuals $I_{h}$, patients treated with TBDs $T_{h}$, successful treatment with TBDs and not transmitting gamete cells to mosquito individuals $P_{h}$, and recovered individuals but partially lost their immunity $R_{h}$. The total mosquito population $N_{v}$ is divided into susceptible mosquitoes $S_{v}$ and infected mosquitoes $I_{v}$. At any time $t$, the human population has the expression $\quad N_{h}(t)=S_{h}(t)+I_{h}(t)+T_{h}(t)+P_{h}(t)+R_{h}(t)$ and the mosquito population has the expression $N_{v}(t)=S_{v}(t)+I_{v}(t)$. The period from the time of human being infected with Plasmodium to the time of onset is recorded as the time delay $\tau$. The model considered in this paper is 


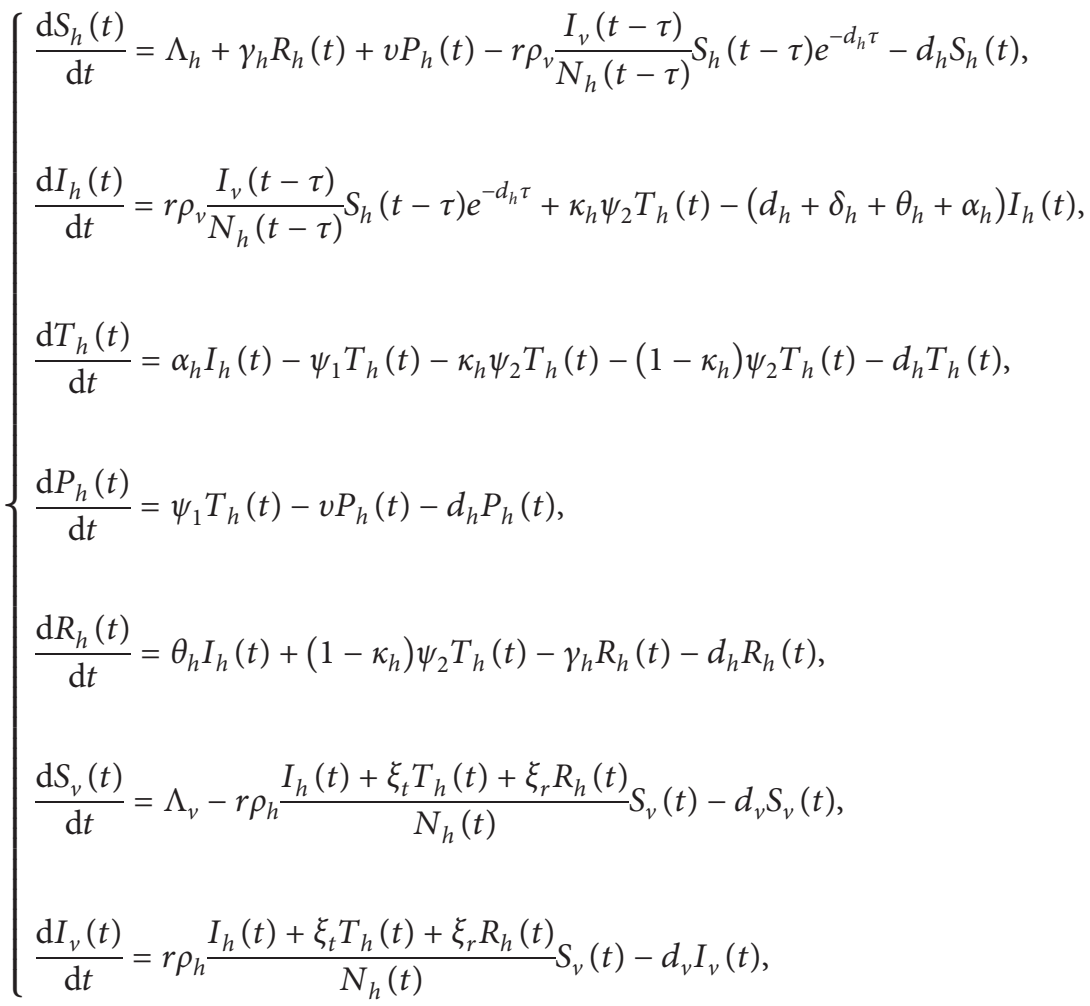

where $\Lambda_{h}$ and $\Lambda_{v}$ are the input of susceptible humans and susceptible mosquitoes, respectively, and $d_{h}$ and $d_{v}$ are the natural mortality rates of humans and mosquitoes, respectively. The exponential term $e^{-d_{h} \tau}$ represents the human survival rate during the incubation period. $\gamma_{h}$ is the immune loss rate after human recovery, and $v$ represents the rate of loss protection and transfer to the susceptible class. $\psi_{1}$ is the percentage of patients who were successfully protected after TBDs treatment, and $\psi_{2}$ is the percentage of patients who were not protected after TBDs treatment. Among the patients who were not protected after TBDs treatment, $\kappa_{h} \psi_{2}$ is the rate of being moved to infection class, and $\left(1-\kappa_{h}\right) \psi_{2}$ is the rate of being moved to the recovery class. $\psi_{1}$ and $\psi_{2}$ are functions of $p_{e}$ ( $p_{e}$ is the efficacy of TBDs), $\psi_{1}$ is proportional to $p_{e}$, and $\psi_{2}$ is inversely proportional to $p_{e}$, respectively, expressed as simple functions $\psi_{1}=\eta_{h} p_{e}$ and $\psi_{2}=\mu_{h}\left(1-p_{e}\right) \cdot \eta_{h}$ is the effective rate of TBDs treatment. $\mu_{h}$ is the rate of ineffective TBDs treatment. $\alpha_{h}$ represents the rate of infected patients receiving TBDs treatment (abbreviated as treatment rate), $\delta_{h}$ is the human mortality rate due to disease, and $\theta_{h}$ is the human recovery rate after infection. $f_{h}=r \rho_{v}\left(I_{v} / N_{h}\right)$ is the human infection rate, where $r$ is the average number of times a mosquito bites a human and $\rho_{v}$ is the probability of human infection for each bite of mosquito. $f_{v}=r \rho_{h}\left(\left(I_{h}+\xi_{t} T_{h}+\xi_{r} R_{h}\right) / N_{h}\right)$ represents the infection rate of mosquitoes, and $\rho_{h}$ represents the probability of mosquito infection per bite. $\xi_{t}$ and $\xi_{r}$ represent the relative infection rates respectively, and $0<\xi_{r}<\xi_{t}<1$. Let $\beta_{1 h}=d_{h}+\delta_{h}+\theta_{h}+\alpha_{h}$, assuming that the input of the susceptible population is greater than the natural human mortality rate $\left(\Lambda_{h}>d_{h}\right)$; otherwise, the human will go extinct.
Let $C=C\left([-\tau, 0], R_{+}^{7}\right)$ be a Banach space of continuous functions mapping the interval $[-\tau, 0]$ into $R_{+}^{7}$ with upper bound norm. According to the biological significance, the initial conditions of system (1) are given

$$
\begin{aligned}
S_{h}(\theta) & =\varphi_{1}(\theta), \\
I_{h}(\theta) & =\varphi_{2}(\theta), \\
T_{h}(\theta) & =\varphi_{3}(\theta), \\
P_{h}(\theta) & =\varphi_{4}(\theta), \\
R_{h}(\theta) & =\varphi_{5}(\theta), \\
I_{v}(\theta) & =\varphi_{6}(\theta), \\
N_{v}(\theta) & =\varphi_{7}(\theta), \\
\theta & \in[-\tau, 0] .
\end{aligned}
$$

Among them

$$
\varphi=\left(\varphi_{1}, \varphi_{2}, \varphi_{3}, \varphi_{4}, \varphi_{5}, \varphi_{6}, \varphi_{7}\right)^{T} \in C^{+},
$$

where

$$
C^{+}=\left\{\varphi=\left(\varphi_{1}, \varphi_{2}, \varphi_{3}, \varphi_{4}, \varphi_{5}, \varphi_{6}, \varphi_{7}\right)^{T} \in C: \varphi_{i} \geq 0, i=1,2, \ldots, 7\right\} .
$$

According to the basic theory of functional differential equation, system (1) has a unique solution which satisfies the initial conditions.

According to the first five equations of system (1), for $t \geq 0$, there is

$$
\dot{N}_{h}=\dot{S}_{h}+\dot{I}_{h}+\dot{T}_{h}+\dot{P}_{h}+\dot{R}_{h}=\Lambda_{h}-d_{h} N_{h}-\delta_{h} I_{h} .
$$


Thus, $\lim _{t \longrightarrow+\infty} \sup _{h}(t) \leq\left(\Lambda_{h} / d_{h}\right) \equiv N_{h \max }$. Similarly, from the last two expressions of system (1), there is $\lim _{t \longrightarrow+\infty} \sup N_{v}(t) \leq\left(\Lambda_{v} / d_{v}\right) \equiv N_{v \max }$. Therefore, the solution of system (1) is ultimately uniformly bounded.

Let

$$
\Omega=\left\{\left(N_{h}, N_{v}\right) \mid 0<N_{h} \leq N_{h \max }, 0<N_{v} \leq N_{v \max }\right\},
$$

and then all trajectories in the first quadrant of system (1) will enter or stay in $\Omega$. Therefore, all variables on $\Omega$ are nonnegative and ultimately uniformly bounded, and the initial value problem of system (1) satisfies the biological significance. Next, the dynamic properties of system (1) are considered on $\Omega$.

In the third section, the control reproduction number and the classification of the equilibrium are calculated, which indicates that the system has a backward bifurcation. In the fourth section, the local stability and global stability of the disease-free equilibrium are discussed. In the fifth section, the influence of TBDs on the propagation of the model is discussed. In the sixth section, the local stability of the endemic equilibrium and the existence of Hopf bifurcation are analyzed. Finally, numerical simulation is used to verify the conclusions obtained in this paper.

\section{The Existence of Equilibrium}

In this section, the equilibria of the model are classified and the control reproduction number of the model is determined.

When there is no infection, the system has a stable state. Supposing that the disease-free equilibrium is $E_{0}=\left(S_{h}^{0}, I_{h}^{0}\right.$, $\left.T_{h}^{0}, P_{h}^{0}, R_{h}^{0}, S_{v}^{0}, I_{v}^{0}\right)$, it is easy to obtain $E_{0}=\left(\left(\Lambda_{h} / d_{h}\right), 0,0,0,0\right.$, $\left.\left(\Lambda_{v} / d_{v}\right), 0\right)$.

When infection exists, the endemic equilibrium $E^{*}=$ $\left(S_{h}^{*}, I_{h}^{*}, T_{h}^{*}, P_{h}^{*}, R_{h}^{*}, S_{v}^{*}, I_{v}^{*}\right)$ of system (1) satisfies the following equations:

$$
\left\{\begin{array}{l}
\Lambda_{h}+\gamma_{h} R_{h}^{*}+v P_{h}^{*}-f_{h}^{*} S_{h}^{*} e^{-d_{h} \tau}-d_{h} S_{h}^{*}=0, \\
f_{h}^{*} S_{h}^{*} e^{-d_{h} \tau}+\kappa_{h} \psi_{2} T_{h}^{*}-\beta_{1 h} I_{h}^{*}=0, \\
\alpha_{h} I_{h}^{*}-\psi_{1} T_{h}^{*}-\kappa_{h} \psi_{2} T_{h}^{*}-\left(1-\kappa_{h}\right) \psi_{2} T_{h}^{*}-d_{h} T_{h}^{*}=0, \\
\psi_{1} T_{h}^{*}-v P_{h}^{*}-d_{h} P_{h}^{*}=0, \\
\theta_{h} I_{h}^{*}+\left(1-\kappa_{h}\right) \psi_{2} T_{h}^{*}-\gamma_{h} R_{h}^{*}-d_{h} R_{h}^{*}=0, \\
\Lambda_{v}-f_{v}^{*} S_{v}^{*}-d_{v} S_{v}^{*}=0 \\
f_{v}^{*} S_{v}^{*}-d_{v} I_{v}^{*}=0 .
\end{array}\right.
$$

From the first five equations, it follows that

$$
\left\{\begin{array}{l}
S_{h}^{*}=\frac{K_{2} I_{h}^{*}}{T_{1} f_{h}^{*} e^{-d_{h} \tau}}, \\
T_{h}^{*}=\frac{\alpha_{h} I_{h}^{*}}{T_{1}}, \\
P_{h}^{*}=\frac{\alpha_{h} \psi_{1} I_{h}^{*}}{T_{1} T_{2}}, \\
R_{h}^{*}=\frac{\theta_{h} T_{1}+\left(1-\kappa_{h}\right) \alpha_{h} \psi_{2}}{T_{1} T_{3}} I_{h}^{*}, \\
I_{h}^{*}=\frac{a_{1} f_{h}}{a_{2} f_{h}+a_{3}},
\end{array}\right.
$$

where

$$
\begin{aligned}
& T_{1}=\psi_{1}+\psi_{2}+d_{h}, \\
& T_{2}=v+d_{h}, \\
& T_{3}=\gamma_{h}+d_{h}, \\
& K_{1}=T_{1} T_{3}+\xi_{t} \alpha_{h} T_{3}+\xi_{r} K_{5}, \\
& K_{2}=\beta_{1 h} T_{1}-\kappa_{h} \alpha_{h} \psi_{2}>0, \\
& K_{3}=\kappa_{h} \psi_{2} \theta_{h}+\left(1-\kappa_{h}\right) \psi_{2} \beta_{1 h}, \\
& K_{4}=\frac{r \rho_{v} \Lambda_{v} d_{h}}{\Lambda_{h} d_{v}}, \\
& K_{5}=\theta_{h} T_{1}+\alpha_{h} \psi_{2}\left(1-\kappa_{h}\right), \\
& a_{1}=\Lambda_{h} T_{1} T_{2} T_{3} e^{-d_{h} \tau}>0, \\
& a_{2}=T_{2} T_{3} K_{2} e^{-d_{h} \tau}-T_{2} \gamma_{h} K_{5} e^{-d_{h} \tau}-v \alpha_{h} \psi_{1} T_{3} e^{-d_{h} \tau}>0, \\
& a_{3}=T_{2} T_{3} d_{h} K_{2}>0 .
\end{aligned}
$$

From the model, there are

$$
\begin{aligned}
& N_{h}^{*}=\frac{\Lambda_{h}-\delta_{h} I_{h}^{*}}{d_{h}}, \\
& N_{v}^{*}=\frac{\Lambda_{v}}{d_{v}} .
\end{aligned}
$$

Substituting the expressions of $I_{h}^{*}, T_{h}^{*}, R_{h}^{*}$, and $N_{h}^{*}$ in (8) and (10) into $f_{v}=r \rho_{h}\left(\left(I_{h}+\xi_{t} T_{h}+\xi_{r} R_{h}\right) / N_{h}\right)$, then 


$$
f_{v}^{*}=\frac{r \rho_{h} d_{h} a_{1} K_{1} f_{h}^{*}}{T_{1} T_{3} b_{1} f_{h}^{*}+T_{1} T_{3} a_{3} \Lambda_{h}},
$$

where $b_{1}=a_{2} \Lambda_{h}-a_{1} \delta_{h}>0$ and $K_{1}=T_{1} T_{3}+\xi_{t} \alpha_{h} T_{3}+$ $\xi_{r} K_{5}>0$.

It can be obtained from the last two equations of (7) that

$$
\begin{aligned}
& S_{v}^{*}=\frac{\Lambda_{v}}{f_{v}^{*}+d_{v}}, \\
& I_{v}^{*}=\frac{\Lambda_{v} f_{v}^{*}}{d_{v}\left(f_{v}^{*}+d_{v}\right)} .
\end{aligned}
$$

$$
\begin{aligned}
l_{1} & =r \rho_{h} d_{h} d_{v} a_{1} b_{1} K_{1}+T_{1} T_{3} d_{v}^{2} b_{1}^{2}, \\
l_{2} & =\frac{\Lambda_{h} d_{h} d_{v}^{2} a_{1} a_{2} K_{2} T_{3}}{e^{-d_{h} \tau}}\left(\frac{e^{-d_{h} \tau}\left(r \rho_{h} d_{h} a_{1} a_{3} K_{1}+2 T_{1} T_{3} d_{v} b_{1} a_{3}\right)}{d_{h} d_{v} a_{1} a_{2} K_{2} T_{3}}-R_{T}\right), \\
l_{3} & =T_{1} T_{3} d_{v}^{2} \Lambda_{h}^{2} a_{3}^{2}\left(1-R_{T}\right), \\
R_{T} & =\frac{r^{2} \rho_{h} \rho_{v} \Lambda_{v} d_{h} K_{1} e^{-d_{h} \tau}}{\Lambda_{h} d_{v}^{2} K_{2} T_{3}} .
\end{aligned}
$$

$R_{T}$ is defined as the control reproduction number of the model.

It follows that the number of positive equilibria of system (1) depends on the number of positive roots of equation (14). For equation (14), there are $F(0)=T_{1} T_{3} d_{v}^{2} \Lambda_{h}^{2} a_{3}^{2}\left(1-R_{T}\right)$ and $l_{1}>0$. When $R_{T}>1$, there are $F(0)<0$ and $\lim _{f^{*} \longrightarrow+\infty} F\left(f_{h}^{*}\right)>0$. Thus, when $R_{T}>1$, equation (14) must have a unique positive root, denoted as $f_{h}^{*}$.

Let

$$
\begin{aligned}
& M=\frac{e^{-d_{h} \tau}\left(r \rho_{h} d_{h} a_{1} a_{3} K_{1}+2 T_{1} T_{3} d_{v} b_{1} a_{3}\right)}{d_{h} d_{v} a_{1} a_{2} K_{2} T_{3}}, \\
& N=\frac{\Lambda_{h} d_{h} d_{v}^{2} a_{1} a_{2} K_{2} T_{3}}{e^{-d_{h} \tau},} \\
& Q=T_{1} T_{3} d_{v}^{2} \Lambda_{h}^{2} a_{3}^{2},
\end{aligned}
$$

and then $M>0, N>0$, and $Q>0$.

When $R_{T}=1$, there are $l_{3}=0$ and $F\left(f_{h}^{*}\right)=l_{1} f_{h}^{* 2}+l_{2} f_{h}^{*}$. Thus, when $l_{2}<0$, namely, $M<1$, equation (14) has a unique positive root.

When $R_{T}<1$, there is $F(0)>0$. If $M>1$, equation (14) has no positive roots; positive roots of equation (14) are possible only when $M<1$. Further, for equation (14) to have positive roots, the condition $M<R_{T}$ needs to be satisfied.

The discriminant of $F\left(f_{h}^{*}\right)=0$ is $\Delta=l_{2}^{2}-4 l_{1} l_{3}$; rearrange it as a function of $R_{T}$ and call it $\Delta\left(R_{T}\right)$.
Substituting the expressions of $I_{v}^{*}$ and $N_{h}^{*}$ in (12) and (10) into $f_{h}=r \rho_{v}\left(I_{v} / N_{h}\right)$, then

$$
f_{h}^{*}=\frac{r \rho_{v} \Lambda_{v} d_{h}\left(a_{2} f_{h}^{*}+a_{3}\right) f_{v}^{*}}{d_{v}\left(f_{v}^{*}+d_{v}\right)\left(b_{1} f_{h}^{*}+a_{3} \Lambda_{h}\right)} .
$$

From (11) and (13), the quadratic equation of one variable about $f_{h}^{*}$ is obtained as follows:

$$
F\left(f_{h}^{*}\right)=l_{1} f_{h}^{* 2}+l_{2} f_{h}^{*}+l_{3}=0
$$

where

Let

$$
R^{*} \triangleq \frac{M N^{2}-2 l_{1} Q+2 \sqrt{l_{1}^{2} Q^{2}+l_{1} N^{2} Q(1-M)}}{N^{2}} .
$$

By simple computations, it has $\Delta(0)>0, \Delta(M)<0$, $\Delta\left(R^{*}\right)=0$, and $\Delta(1)>0$. Thus, $0<M<R^{*}<1$.

If $R^{*}<R_{T}<1$, equation (14) has two positive roots, denoted as $f_{h}^{*}$ and $f_{h}^{* *}$. If $R_{T}=R^{*}$, equation (14) has a positive root, denoted as $f_{h}^{\prime}$. If $0<R_{T}<R^{*}$, equation (14) has no positive root. Based on the above analysis, the following theorem can be obtained.

Theorem 1. System (1) always has a disease-free equilibrium $E_{0}=\left(\left(\Lambda_{h} / d_{h}\right), 0,0,0,0,\left(\Lambda_{v} / d_{v}\right), 0\right)$. In addition, system (1) also has the following endemic equilibrium:

(i) If $R_{T}>1$, system (1) has a unique positive equilibrium $E^{*}=\left(S_{h}^{*}, I_{h}^{*}, T_{h}^{*}, P_{h}^{*}, R_{h}^{*}, S_{v}^{*}, I_{v}^{*}\right)$

(ii) If $M<1$, there are three situations:

(1) If $R_{T}=1$, system (1) has a unique positive equilibrium $E^{*}=\left(S_{h}^{*}, I_{h}^{*}, T_{h}^{*}, P_{h}^{*}, R_{h}^{*}, S_{v}^{*}, I_{v}^{*}\right)$

(2) If $R^{*}<R_{T}<1$, system (1) has two positive equilibria $E^{*}=\left(S_{h}^{*}, I_{h}^{*}, T_{h}^{*}, P_{h}^{*}, R_{h}^{*}, S_{v}^{*}, I_{v}^{*}\right) \quad$ and $E^{* *}=\left(S_{h}^{* *}, I_{h}^{* *}, T_{h}^{* *}, P_{h}^{* *}, R_{h}^{* *}, S_{v}^{* *}, T_{v}^{* *}\right)$

(3) If $R_{T}=R^{*}$, system (1) has a unique positive equilibrium $E^{\prime}=\left(S_{h}^{\prime}, I_{h}^{\prime}, T_{h}^{\prime}, P_{h}^{\prime}, R_{h}^{\prime}, S_{v}^{\prime}, I_{v}^{\prime}\right)$

The positive roots of equation (14) are expressed as 


$$
\begin{gathered}
f_{h}^{*}=\frac{-l_{2}+\sqrt{\Delta}}{2 l_{1}}, \\
f_{h}^{* *}=\frac{-l_{2}-\sqrt{\Delta}}{2 l_{1}}, \\
f_{h}^{\prime}=\frac{-l_{2}}{2 l_{1}} .
\end{gathered}
$$

\section{Stability of the Disease-Free Equilibrium}

In this section, the local stability of the disease-free equilibrium is discussed by using the characteristic equation of the linear approximation equation of system (1). In addition, the global stability of the disease-free equilibrium is obtained by constructing the Lyapunov function when the time delay is zero.

4.1. Local Stability of the Disease-Free Equilibrium. For the local stability of the disease-free equilibrium $E_{0}$, the following conclusions are obtained.

Theorem 2. If $R_{T}<1$, the disease-free equilibrium $E_{0}$ is locally asymptotically stable for any $\tau \geq 0$; if $R_{T}>1, E_{0}$ is unstable for any $\tau \geq 0$.

Proof. It is shown that the characteristic equation of system (1) at $E_{0}$ is

$$
L_{0}(\lambda, \tau)=\lambda^{4}+A_{3} \lambda^{3}+A_{2} \lambda^{2}+A_{1} \lambda+A_{0}+\mathrm{e}^{-\lambda \tau}\left(B_{2} \lambda^{2}+B_{1} \lambda+B_{0}\right)=0,
$$

where

$$
\begin{aligned}
& A_{3}=T_{1}+T_{3}+d_{v}+\beta_{1 h}, \\
& A_{2}=K_{2}+d_{v} T_{3}+\left(T_{3}+d_{v}\right)+\left(T_{1}+\beta_{1 h}\right), \\
& A_{1}=d_{v} T_{3}\left(T_{1}+\beta_{1 h}\right)+\left(T_{3}+d_{v}\right) K_{2}, \\
& A_{0}=d_{v} T_{3} K_{2}, \\
& B_{2}=-r \rho_{v} K_{4} e^{-d_{h} \tau}, \\
& B_{1}=-r \rho_{v} K_{4}\left(T_{1}+T_{3}+\theta_{h} \xi_{r}+\alpha_{h} \xi_{t}\right) e^{-d_{h} \tau}, \\
& B_{0}=-r \rho_{v} K_{4} K_{1} e^{-d_{h} \tau} .
\end{aligned}
$$

When $\tau=0$, equation (19) is rewritten as

$$
L_{1}(\lambda)=\lambda^{4}+m_{3} \lambda^{3}+m_{2} \lambda^{2}+m_{1} \lambda+m_{0}=0,
$$

where $m_{3}=A_{3}, \quad m_{2}=A_{2}+B_{2}, \quad m_{1}=A_{1}+B_{1}$, and $m_{0}=A_{0}+B_{0}$.

When $R_{T}<1$, there are

$$
\begin{aligned}
& m_{3}=A_{3}>0 \\
& m_{2}=A_{2}+B_{2}>K_{2}+d_{v}\left(T_{1}+T_{3}\right)+T_{3}\left(T_{1}+\beta_{1 h}\right)>0 \\
& m_{1}=A_{1}+B_{1}>d_{v} T_{1} T_{3}+T_{3} K_{2}>0 \\
& m_{0}=A_{0}+B_{0}=d_{v} T_{3} K_{2}\left(1-R_{T}\right)>0 .
\end{aligned}
$$

Next, consider the signs of $\Delta_{2}=m_{3} m_{2}-m_{1}$ and $\Delta_{3}=m_{1}\left(m_{3} m_{2}-m_{1}\right)-m_{0} m_{3}^{2}$.

$$
\begin{aligned}
\Delta_{2}= & m_{3} m_{2}-m_{1} \\
> & a_{3}\left[d_{v} T_{1}+T_{3}\left(T_{1}+\beta_{1 h}\right)\right]+\left(T_{1}+\beta_{1 h}\right) K_{2} \\
& +\left(d_{v}+T_{3}\right) d_{v} T_{3}+r \rho_{v} K_{4}\left(T_{1}+T_{3}+\theta_{h} \xi_{r}+\alpha_{h} \xi_{t}\right)>0, \\
\Delta_{3}= & m_{1}\left(m_{3} m_{2}-m_{1}\right)-m_{0} m_{3}^{2} \\
> & \left(T_{3} K_{2}+d_{v} T_{1} T_{3}\right)\left[K_{2}\left(T_{1}+\beta_{1 h}\right)+d_{v} T_{3}\left(d_{v}+T_{3}\right)\right] \\
& +T_{3}^{2} K_{2} a_{3}\left(T_{1}+\beta_{1 h}\right)+d_{v} T_{1}^{2} T_{3} a_{3}\left(d_{v}+T_{3}\right)+r \rho_{v} K_{4} a_{3} T_{1} T_{3}\left(T_{1}+T_{3}\right)>0 .
\end{aligned}
$$

According to the Routh-Hurwitz criterion, when $\tau=0$ and $R_{T}<1$, the disease-free equilibrium $E_{0}$ is locally asymptotically stable. Next, the local stability of disease-free equilibrium $E_{0}$ is discussed when $\tau>0$. Suppose that, for some $\tau>0$, equation (19) has a pure imaginary root $\lambda=i \omega$, $\omega>0$. Substituting $\lambda=i \omega$ into equation (19), then equation (19) becomes

$$
\begin{gathered}
\omega^{4}-A_{3} \omega^{3} i-A_{2} \omega^{2}+A_{1} \omega i+A_{0}+(\cos \omega \tau-i \sin \omega \tau) \\
\cdot\left(-B_{2} \omega^{2}+B_{1} \omega i+B_{0}\right)=0 .
\end{gathered}
$$

Separating the real part and the imaginary part gives

$$
\left\{\begin{array}{l}
\left(-B_{2} \omega^{2}+B_{0}\right) \cos \omega \tau+B_{1} \omega \sin \omega \tau=-\omega^{4}+A_{2} \omega^{2}-A_{0} \\
B_{1} \omega \cos \omega \tau-\left(-B_{2} \omega^{2}+B_{0}\right) \sin \omega \tau=A_{3} \omega^{3}-A_{1} \omega
\end{array}\right.
$$

Then $\omega$ must be the root of the following equation:

$$
\omega^{8}+c_{3} \omega^{6}+c_{2} \omega^{4}+c_{1} \omega^{2}+c_{0}=0 .
$$

Let $z=\omega^{2}$; then equation (26) becomes the following equation:

$$
z^{4}+c_{3} z^{3}+c_{2} z^{2}+c_{1} z+c_{0}=0
$$

where 


$$
\begin{aligned}
& c_{3}=A_{3}^{2}-2 A_{2}, \\
& c_{2}=A_{2}^{2}+2 A_{0}-2 A_{1} A_{3}-B_{2}^{2}, \\
& c_{1}=A_{1}^{2}-2 A_{0} A_{2}-B_{1}^{2}+2 B_{0} B_{2}, \\
& c_{0}=A_{0}^{2}-B_{0}^{2} .
\end{aligned}
$$

$$
\begin{aligned}
c_{0}= & d_{v}^{2} T_{3}^{2} K_{2}^{2}\left(1-R_{T}^{2}\right)>0, \\
c_{1}> & d_{v}^{2} T_{1}^{2} T_{3}^{2}+k_{2}^{2} T_{3}^{2}+2 r^{2} \rho_{v}^{2} K_{4}^{2} \xi_{r} \alpha_{h} \psi_{2}\left(1-\kappa_{h}\right) \\
& +2 d_{v}^{2} T_{3}^{2} \kappa_{h} \alpha_{h} \psi_{2}>0, \\
c_{2}> & K_{2}^{2}+d_{v}^{2}\left(T_{3}^{2}+T_{1}^{2}\right)+T_{3}^{2}\left(T_{1}^{2}+\beta_{1 h}^{2}\right)>0, \\
c_{3}> & T_{1}^{2}+T_{3}^{2}+\beta_{1 h}^{2}+d_{v}^{2}>0 .
\end{aligned}
$$

Therefore, when $R_{T}<1$, equation (27) has no positive root.

According to the above analysis, when $R_{T}<1$, all the roots of the characteristic equation (19) have negative real parts, and the disease-free equilibrium $E_{0}$ is locally asymptotically stable. When $R_{T}>1$, there are $L_{0}(0, \tau)=d_{v}$ $T_{3} K_{2}\left(1-R_{T}\right)<0$ and $\lim _{\lambda \longrightarrow+\infty} L_{0}(\lambda, \tau)>0$, the characteristic equation (19) has at least one positive real root, and then the disease-free equilibrium $E_{0}$ is unstable.
4.2. Global Stability of the Disease-Free Equilibrium. From the above discussion, the disease-free equilibrium $E_{0}$ is locally asymptotically stable when $R_{T}<1$. In this section, the global stability of disease-free equilibrium $E_{0}$ is considered at $\tau=0$.

Let

$$
\begin{aligned}
& \widetilde{R}=\frac{d_{h}^{2} K_{1}}{\Lambda_{h} K_{2} T_{3}}, \\
& \Gamma=\left\{\left(S_{h}, I_{h}, T_{h}, P_{h}, R_{h}, S_{v}, I_{v}\right) \in R_{+}^{7}: 0<S_{h}+I_{h}+T_{h}+P_{h}+R_{h} \leq \frac{\Lambda_{h}}{d_{h}} ; 0<S_{v}+I_{v} \leq \frac{\Lambda_{v}}{d_{v}}\right\} .
\end{aligned}
$$

Theorem 3. If $R_{T}<\widetilde{R}$, the disease-free equilibrium $E_{0}$ is globally asymptotically stable in $\Gamma$ at $\tau=0$.

Proof. It can be seen from the above section that the diseasefree equilibrium $E_{0}$ is locally asymptotically stable. Next, only the global attraction of the disease-free equilibrium $E_{0}$ is required to be proved.

By calculation, there is

$$
\widetilde{R}=\frac{d_{h}^{2} K_{1}}{\Lambda_{h} K_{2} T_{3}} \leq \frac{d_{h}^{2} K_{1}}{\Lambda_{h} d_{h} K_{1}+K_{6}}<1,
$$

where $K_{6}=\Lambda_{h}\left[\delta_{h} T_{1} T_{3}+\theta_{h} T_{1} \gamma_{h}+\alpha_{h} \psi_{1} T_{3}+\alpha_{h} \psi_{2}\left(1-\kappa_{h}\right)\right.$ $\left.\gamma_{h}\right]>0$.

Constructing the Lyapunov function on $\Gamma$,

$$
V=\frac{r \rho_{h} \Lambda_{v}}{d_{h} d_{v}} I_{h}+\frac{r \rho_{h} \Lambda_{v}}{d_{h} d_{v}} T_{h}+\frac{r \rho_{h} \Lambda_{v}}{d_{h} d_{v}} R_{h}+I_{v} .
$$

$V(t)$ is positive and bounded according to the nonnegative uniform boundedness of the solution. The total derivative of $V(t)$ along the solution $x_{t}$ of system (1) is 


$$
\begin{aligned}
\dot{V}\left(x_{t}\right)= & \frac{r \rho_{h} \Lambda_{v}}{d_{h} d_{v}} \dot{I}_{h}+\frac{r \rho_{h} \Lambda_{v}}{d_{h} d_{v}} \dot{T}_{h}+\frac{r \rho_{h} \Lambda_{v}}{d_{h} d_{v}} \dot{R}_{h}+\dot{I}_{v} \\
= & \frac{r \rho_{h} \Lambda_{v}}{d_{h} d_{v}}\left(r \rho_{v} \frac{I_{v}}{N_{h}} S_{h} e^{-d_{h} \tau}+\kappa_{h} \psi_{2} T_{h}-\beta_{1 h} I_{h}+\alpha_{h} I_{h}-\psi_{1} T_{h}-\psi_{2} T_{h}-d_{h} T_{h}\right) \\
& +\frac{r \rho_{h} \Lambda_{v}}{d_{h} d_{v}}\left[\theta_{h} I_{h}+\left(1-\kappa_{h}\right) \psi_{2} T_{h}-\gamma_{h} R_{h}-d_{h} R_{h}\right]+r \rho_{h} \frac{I_{h}+\xi_{t} T_{h}+\xi_{r} R_{h}}{N_{h}}-d_{v} I_{v} \\
\leq & \frac{r \rho_{h} \Lambda_{v}}{d_{h} d_{v}}\left[r \rho_{v} I_{v} e^{-d_{h} \tau}-\left(d_{h}+\delta_{h}\right) I_{h}-\psi_{1} T_{h}-d_{h} T_{h}-\gamma_{h} R_{h}-d_{h} R_{h}\right] \\
& +\frac{r \rho_{h} \Lambda_{v}}{d_{v}}\left(I_{h}+T_{h}+R_{h}\right)-d_{v} I_{v} \\
\leq & d_{v}\left(\frac{R_{T}}{\widetilde{R}}-1\right) I_{v}-\frac{r \rho_{h} \Lambda_{v}}{d_{h} d_{v}}\left(\delta_{h} I_{h}+\psi_{1} T_{h}+\gamma_{h} R_{h}\right) .
\end{aligned}
$$

When $R_{T}<\widetilde{R}$, there is $\dot{V}\left(x_{t}\right) \leq 0$, if and only if $I_{v}=0, I_{h}=0$, $T_{h}=0$, and $R_{h}=0$, and there is $\dot{V}\left(x_{t}\right)=0$. Define the set

$$
\Sigma=\left\{\left(S_{h}, I_{h}, T_{h}, P_{h}, R_{h}, S_{v}, I_{v}\right) \in \Gamma \mid \frac{\mathrm{d} V}{\mathrm{~d} t}=0\right\} .
$$

Let $M$ be the largest invariant set in $\Sigma$. From system (1) and the invariant property of $M, M=\left\{E_{0}\right\}$ can be obtained. According to the Lyapunov-LaSalle principle, the solutions of system (1) with initial conditions in $\Gamma$ all approach the disease-free equilibrium $E_{0}$ at $t \longrightarrow+\infty$. That is, when $R_{T}<\widetilde{R}$, there is $\left(S_{h}(t), I_{h}(t), T_{h}(t), P_{h}(t), \quad R_{h}(t), S_{v}(t)\right.$, $\left.I_{v}(t)\right) \longrightarrow\left(\left(\Lambda_{h} / d_{h}\right), 0,0,0,0,\left(\Lambda_{v} / d_{v}\right), 0\right)$; the disease-free equilibrium $E_{0}$ is globally asymptotically stable.

\section{Assessment of TBDs}

In order to effectively control malaria for a long time, it is necessary to know what conditions the parameters meet when the use of TBDs plays a positive role in disease control. In this section, the impact of the use of TBDs on malaria transmission is analyzed. Firstly, the treatment rate $\alpha_{h}$ was taken as the main parameter to consider the influence of $\alpha_{h}$ on the control reproduction number $R_{T}$.

Let

$$
\begin{aligned}
& c_{0}=r^{2} \rho_{h} \rho_{v} \Lambda_{v} d_{h} e^{-d_{h} \tau}, \\
& c_{1}=T_{1}\left(T_{3}+\xi_{r} \theta_{h}\right), \\
& c_{2}=T_{1}\left(d_{h}+\delta_{h}+\theta_{h}\right), \\
& d_{0}=T_{3} \Lambda_{h} d_{v}^{2}, \\
& d_{1}=\xi_{t} T_{3}+\xi_{r} \psi_{2}\left(1-\kappa_{h}\right), \\
& d_{2}=T_{1}-\kappa_{h} \psi_{2} .
\end{aligned}
$$

The derivative of $R_{T}$ with respect to $\alpha_{h}$ is

$$
\frac{\mathrm{d} R_{T}}{\mathrm{~d} \alpha_{h}}=\frac{c_{0}\left(c_{2} d_{1}-c_{1} d_{2}\right)}{d_{0}\left(c_{2}+d_{2} \alpha_{h}\right)^{2}},
$$

if and only if $c_{2} d_{1}-c_{1} d_{2}<0$, and there is $\left(\mathrm{d} R_{T} / \mathrm{d} \alpha_{h}\right)<0$. In general, when the control reproduction number is less than one, the disease can finally disappear, so there is the following theorem.

Theorem 4. For system (1),

(1) When $\left(c_{2} d_{1} / c_{1} d_{2}\right)<1$, the TBDs have a positive effect on reducing the burden of disease

(2) When $\left(c_{2} d_{1} / c_{1} d_{2}\right)=1$, the TBDs have no effect on reducing the burden of disease

(3) When $\left(c_{2} d_{1} / c_{1} d_{2}\right)>1$, the TBDs have a negative effect on reducing the burden of disease

It is generally believed that the more the people who receive the TBDs treatment, the more the effectiveness on controlling malaria. However, humans treated with TBDs remain infectious; the increase in the number of $T_{h}$ does not control the disease well. It is worth noting the percentage of humans treated with TBDs who were successfully protected. Next, taking the efficacy of TBDs $p_{e}$ as the main parameter, to observe which conditions the parameters meet, the TBDs have a positive effect on reducing the burden of disease.

The expression $c_{2} d_{1}-c_{1} d_{2}$ is rewritten as the expression $T_{1}\left(E_{1}+E_{2} p_{e}\right)$ about the efficacy of TBDs $p_{e}$, where

$$
\begin{aligned}
& E_{1}=\left(d_{h}+\delta_{h}+\theta_{h}\right) \xi_{r} T_{3}-d_{h} T_{3}-d_{h} \theta_{h} \xi_{r}-\left(1-\kappa_{h}\right) T_{3} \mu_{h}+\left(d_{h}+\delta_{h}\right) \xi_{r}\left(1-\kappa_{h}\right) \mu_{h}, \\
& E_{2}=\left(1-\kappa_{h}\right) T_{3} \mu_{h}-\left(T_{3}+\theta_{h} \xi_{r}\right) \eta_{h}-\left(d_{h}+\delta_{h}\right) \xi_{r}\left(1-\kappa_{h}\right) \mu_{h} .
\end{aligned}
$$


According to Theorem 4 and equation (36), the following theorem can be obtained.

\section{Theorem 5. For system (1),}

(1) When $E_{1}>0$ and $E_{2}>0$, for any $p_{e} \in[0,1]$, the TBDs have a negative effect on reducing the burden of disease

(2) When $E_{1}>0$ and $E_{2}<0$, if $p_{e} \in\left[\min \left(\left(-E_{1} / E_{2}\right)\right.\right.$, $1), 1]$, the TBDs have a positive effect on reducing the burden of disease
(3) When $E_{1}<0$ and $E_{2}>0$, if $p_{e} \in[0$, min $\left.\left(\left(-E_{1} / E_{2}\right), 1\right)\right]$, the TBDs have a positive effect on reducing the burden of disease

(4) When $E_{1}<0$ and $E_{2}<0$, for any $p_{e} \in[0,1]$, the TBDs have a positive effect on reducing the burden of disease

\section{Stability of Positive Equilibrium and Hopf Bifurcation}

6.1. Local Stability of Positive Equilibrium. When $R_{T}>1$, there is a unique positive equilibrium in system (1), and the characteristic equation of system (1) at $E^{*}$ is

$$
\begin{aligned}
L_{2}(\lambda, \tau)= & \lambda^{7}+F_{6} \lambda^{6}+F_{5} \lambda^{5}+F_{4} \lambda^{4}+F_{3} \lambda^{3}+F_{2} \lambda^{2}+F_{1} \lambda+F_{0} \\
& +e^{-\lambda \tau}\left(J_{6} \lambda^{6}+J_{5} \lambda^{5}+J_{4} \lambda^{4}+J_{3} \lambda^{3}+J_{2} \lambda^{2}+J_{1} \lambda+J_{0}\right)=0 .
\end{aligned}
$$

The coefficients of equation (38) are given in Appendix. When $\tau=0$, the characteristic equation is written as

$$
L_{3}(\lambda)=\lambda^{7}+H_{6} \lambda^{6}+H_{5} \lambda^{5}+H_{4} \lambda^{4}+H_{3} \lambda^{3}+H_{2} \lambda^{2}+H_{1} \lambda+H_{0}=0,
$$

where $H_{i}=F_{i}+J_{i}, i=1,2, \ldots, 6$.

Define

$$
\begin{aligned}
& \Delta_{1}=H_{6} \text {, } \\
& \Delta_{2}=\left|\begin{array}{cc}
H_{6} & 1 \\
H_{4} & H_{5}
\end{array}\right| \text {, } \\
& \Delta_{3}=\left|\begin{array}{ccc}
H_{6} & 1 & 0 \\
H_{4} & H_{5} & H_{6} \\
H_{2} & H_{3} & H_{4}
\end{array}\right| \text {, } \\
& \Delta_{4}=\left|\begin{array}{cccc}
H_{6} & 1 & 0 & 0 \\
H_{4} & H_{5} & H_{6} & 1 \\
H_{2} & H_{3} & H_{4} & H_{5} \\
H_{0} & H_{1} & H_{2} & H_{3}
\end{array}\right|, \\
& \Delta_{5}=\left|\begin{array}{ccccc}
H_{6} & 1 & 0 & 0 & 0 \\
H_{4} & H_{5} & H_{6} & 1 & 0 \\
H_{2} & H_{3} & H_{4} & H_{5} & H_{6} \\
H_{0} & H_{1} & H_{2} & H_{3} & H_{4} \\
0 & 0 & H_{0} & H_{1} & H_{2}
\end{array}\right|, \\
& \Delta_{6}=\left|\begin{array}{cccccc}
H_{6} & 1 & 0 & 0 & 0 & 0 \\
H_{4} & H_{5} & H_{6} & 1 & 0 & 0 \\
H_{2} & H_{3} & H_{4} & H_{5} & H_{6} & 1 \\
H_{0} & H_{1} & H_{2} & H_{3} & H_{4} & H_{5} \\
0 & 0 & H_{0} & H_{1} & H_{2} & H_{3} \\
0 & 0 & 0 & 0 & H_{0} & H_{1}
\end{array}\right| \text {, }
\end{aligned}
$$$$
\Delta_{7}=H_{0} \Delta_{6} \text {. }
$$

According to the Routh-Hurwitz criterion, if $\Delta_{i}>0, i=1,2, \ldots, 7$, then all the roots of equation (39) have negative real parts.

Theorem 6. When $R_{T}>1$ and $\tau=0$, if $\Delta_{i}>0, i=1,2, \ldots, 7$, then the positive equilibrium $E^{*}$ is locally asymptotically stable.

In the classification of equilibria in section three, the occurrence of backward bifurcation means that there are two positive equilibria in the system at $R^{*}<R_{T}<1$. The stability analysis of these two positive equilibria will be explained in numerical simulation.

6.2. Existence of Hopf Bifurcation. Suppose that equation (38) has a pure imaginary $\operatorname{root} \lambda=i \omega(\omega>0) . \lambda=0$ is not the root of equation (38) since $A_{0}+B_{0} \neq 0$. Substituting $\lambda=i \omega$ into equation (38) and separating real and imaginary parts, it follows that

$$
\left\{\begin{array}{l}
p_{1} \cos \omega \tau+p_{2} \sin \omega \tau=F_{6} \omega^{6}-F_{4} \omega^{4}+F_{2} \omega^{2}-F_{0} \\
p_{2} \cos \omega \tau-p_{1} \sin \omega \tau=\omega^{7}-F_{5} \omega^{5}+F_{3} \omega^{3}-F_{1} \omega
\end{array}\right.
$$

where $p_{1}=-J_{6} \omega^{6}+J_{4} \omega^{4}-J_{2} \omega^{2}+J_{0}$ and $p_{2}=J_{5} \omega^{5}-$ $J_{3} \omega^{3}+J_{1} \omega$.

Then $\omega$ satisfies the equation

$$
\begin{aligned}
& \omega^{14}+H_{6} \omega^{12}+H_{5} \omega^{10}+H_{4} \omega^{8}+H_{3} \omega^{6}+H_{2} \omega^{4} \\
& +H_{1} \omega^{2}+H_{0}=0,
\end{aligned}
$$




$$
\begin{aligned}
& H_{6}=F_{6}^{2}-J_{6}^{2}-2 F_{5}, \\
& H_{5}=F_{5}^{2}+2 F_{3}-2 F_{4} F_{6}+2 J_{4} J_{6}-J_{5}^{2}, \\
& H_{4}=F_{4}^{2}+2 F_{2} F_{6}-2 F_{1}-2 F_{3} F_{5}-J_{4}^{2}-2 J_{2} J_{6}+2 J_{3} J_{5}, \\
& H_{3}=F_{3}^{2}+2 F_{1} F_{5}-2 F_{0} F_{6}-2 F_{2} F_{4}-J_{3}^{2}+2 J_{0} J_{6}+2 J_{2} J_{4}-2 J_{1} J_{5}, \\
& H_{2}=F_{2}^{2}+2 F_{0} F_{4}-2 F_{1} F_{3}-J_{2}^{2}-2 J_{0} J_{4}+2 J_{1} J_{3}, \\
& H_{1}=F_{1}^{2}-2 F_{0} F_{2}-J_{1}^{2}+2 J_{0} J_{2}, \\
& H_{0}=F_{0}^{2}-J_{0}^{2} .
\end{aligned}
$$

Let $z=\omega^{2}$, and define

$$
\begin{aligned}
G(z)= & z^{7}+H_{6} z^{6}+H_{5} z^{5}+H_{4} z^{4}+H_{3} z^{3} \\
& +H_{2} z^{2}+H_{1} z+H_{0}=0 .
\end{aligned}
$$

Therefore, if equation (38) has a pure imaginary root, then equation $G(z)=0$ has a positive real root. Assume that equation (44) has seven positive real roots, denoted as $z_{k}, k=1,2, \ldots, 7$.

It can be obtained by calculation that

$$
\left\{\begin{array}{l}
\cos \sqrt{z_{k}} \tau=U_{k}, \\
\sin \sqrt{z_{k}} \tau=V_{k},
\end{array}\right.
$$

where

$$
\begin{aligned}
& U_{k} \triangleq \frac{p_{3}\left(F_{6} z_{k}^{3}-F_{4} z_{k}^{2}+F_{2} z_{k}-F_{0}\right)+z_{k} p_{4}\left(z_{k}^{3}-F_{5} z_{k}^{2}+F_{3} z_{k}-F_{1}\right)}{p_{3}^{2}+z_{k} p_{4}^{2}}, \\
& V_{k} \triangleq \frac{\sqrt{z_{k}} p_{4}\left(F_{6} z_{k}^{3}-F_{4} z_{k}^{2}+F_{2} z_{k}-F_{0}\right)-\sqrt{z_{k}} p_{3}\left(z_{k}^{3}-F_{5} z_{k}^{2}+F_{3} z_{k}-F_{1}\right)}{p_{3}^{2}+z_{k} p_{4}^{2}}, \\
& p_{3}=-J_{6} z_{k}^{3}+J_{4} z_{k}^{2}-J_{2} z_{k}+J_{0}, \\
& p_{4}=J_{5} z_{k}^{2}-J_{3} z_{k}+J_{1} .
\end{aligned}
$$

Then

$$
\tau_{k}^{(j)}= \begin{cases}\frac{1}{\sqrt{y_{k}}}\left[\arccos U_{k}+2 j \pi\right], & V_{k} \geq 0, \\ \frac{1}{\sqrt{y_{k}}}\left[2 \pi-\arccos U_{k}+2 j \pi\right], & V_{k}<0,\end{cases}
$$

where $k=1,2, \ldots, 7$ and $j=0,1,2, \ldots$. Therefore, the characteristic equation $L_{2}\left(\lambda, \tau_{k}^{(j)}\right)=0$ has a pair of pure imaginary roots $\pm i \sqrt{z_{k}}$.

Let

$$
\begin{aligned}
& \tau_{0}=\tau_{k_{0}}^{(0)}=\min \left\{\tau_{k}^{(j)} \mid k=1,2, \ldots, 7 ; j=0,1,2, \ldots\right\}, \\
& \omega_{0}=\omega_{k_{0}} .
\end{aligned}
$$

Assuming that $\lambda(\tau)=\alpha(\tau)+i \omega(\tau)$ is the root of equation (38), then $\alpha\left(\tau_{0}\right)=0$ and $\omega\left(\tau_{0}\right)=\omega_{0}$.

Theorem 7. Suppose that equation (44) has root $z=\omega_{0}^{2}$ and $G^{\prime}(z) \neq 0$; then $\left.(d \operatorname{Re}(\lambda) / d \tau)\right|_{\tau=\tau_{0}}$ and $G^{\prime}(z)$ have the same sign.

Proof. Firstly, the derivative of $\lambda$ with respect to $\tau$ in the characteristic equation (38) is calculated as follows:

$$
\begin{aligned}
\left(\frac{\mathrm{d} \lambda}{\mathrm{d} \tau}\right)^{-1}= & -\frac{7 \lambda^{6}+6 F_{6} \lambda^{5}+5 F_{5} \lambda^{4}+4 F_{4} \lambda^{3}+3 F_{3} \lambda^{2}+2 F_{2} \lambda+F_{1}}{\lambda\left(\lambda^{7}+F_{6} \lambda^{6}+F_{5} \lambda^{5}+F_{4} \lambda^{4}+F_{3} \lambda^{3}+F_{2} \lambda^{2}+F_{1} \lambda+F_{0}\right)} \\
& +\frac{6 J_{6} \lambda^{5}+5 J_{5} \lambda^{4}+4 J_{4} \lambda^{3}+3 J_{3} \lambda^{2}+2 J_{2} \lambda+J_{1}}{\lambda\left(J_{6} \lambda^{6}+J_{5} \lambda^{5}+J_{4} \lambda^{4}+J_{3} \lambda^{3}+J_{2} \lambda^{2}+J_{1} \lambda+J_{0}\right)}-\frac{\tau}{\lambda}
\end{aligned}
$$


TABLE 1: Parameter value range.

\begin{tabular}{|c|c|c|c|c|}
\hline Parameter & Range of possible values & Baseline value used & Dim & Reference \\
\hline$\overline{\Lambda_{h}}$ & {$\left[2.7 \times 10^{-5}, 1000\right]$} & 100 & $H \times$ day $^{-1}$ & Estimated \\
\hline$\Lambda_{v}^{n}$ & {$[0.002,2200]$} & 200 & $V \times$ day $^{-1}$ & Estimated \\
\hline$d_{h}^{v}$ & {$\left[2.74 \times 10^{-5}, 0.033\right]$} & 0.012 & day $^{-1}$ & See [24] \\
\hline$d_{v}$ & {$[0.03302,0.1]$} & 0.08 & day $^{-1}$ & See $[24]$ \\
\hline$\gamma_{h}$ & {$\left[5 \times 10^{-4}, 1\right]$} & 0.01 & day $^{-1}$ & Estimated \\
\hline$v$ & {$[0,0.1]$} & 0.01 & day $^{-1}$ & See $[24]$ \\
\hline$p_{e}$ & {$[0,1]$} & 0.5 & 1 & See $[24]$ \\
\hline$\alpha_{h}$ & {$[0,1]$} & 0.75 & day $^{-1}$ & See $[24]$ \\
\hline$\delta_{h}^{n}$ & {$\left[1 \times 10^{-5}, 5 \times 10^{-2}\right]$} & 0.08 & day $^{-1}$ & See $[24]$ \\
\hline$\theta_{h}$ & {$\left[3.5 \times 10^{-5}, 0.2\right]$} & 0.2 & day $^{-1}$ & See $[24]$ \\
\hline$r$ & {$[0.1,30]$} & 5 & $H \times V^{-1} \times$ day $^{-1}$ & See $[24]$ \\
\hline$\rho_{h}$ & {$[0.01,1]$} & 0.003 & 1 & See $[24]$ \\
\hline$\rho_{v}$ & {$[0.072,1]$} & 0.24 & 1 & See [24] \\
\hline$\xi_{t}$ & {$[0.02,1]$} & 0.4 & 1 & See [24] \\
\hline$\xi_{r}$ & {$[0.005,1]$} & 0.2 & 1 & See $[24]$ \\
\hline$\eta_{h}$ & {$[0,0.2]$} & 0.1 & day $^{-1}$ & See [24] \\
\hline$\mu_{h}$ & {$[0,0.2]$} & 0.2 & day $^{-1}$ & See [24] \\
\hline$\kappa_{h}$ & {$[0,1]$} & 0.6 & day $^{-1}$ & See [24] \\
\hline
\end{tabular}

Substituting $\lambda=i \omega_{0}$ into equation (49), it follows that

$$
\operatorname{Re}\left[\left.\left(\frac{\mathrm{d} \lambda}{\mathrm{d} \tau}\right)^{-1}\right|_{\tau=\tau_{0}}\right]=\frac{G^{\prime}\left(\omega_{0}^{2}\right)}{\left(J_{6} \omega_{0}^{6}-J_{4} \omega_{0}^{4}-J_{2} \omega_{0}^{2}-J_{0}\right)^{2}+\left(J_{5} \omega_{0}^{5}-J_{3} \omega_{0}^{3}-J_{1} \omega_{0}\right)^{2}}
$$

Therefore, it can be obtained that

$$
\begin{aligned}
\operatorname{sign}\left\{\left.\frac{d \operatorname{Re}(\lambda)}{d \tau}\right|_{\tau=\tau_{0}}\right\} & =\operatorname{sign}\left\{\operatorname{Re}\left[\left.\left(\frac{\mathrm{d} \lambda}{\mathrm{d} \tau}\right)^{-1}\right|_{\tau=\tau_{0}}\right]\right\} \\
& =\operatorname{sign}\left\{G^{\prime}\left(\omega_{0}^{2}\right)\right\} .
\end{aligned}
$$

Based on Theorem 7 and Hopf bifurcation theory, the following conclusions can be drawn.

Theorem 8. Suppose that $E^{*}$ is locally asymptotically stable when $\tau=0$ and $R_{T}>1$; there are the following conclusions:

(1) If equation (44) has no positive real root, then the equilibrium $E^{*}$ is locally asymptotically stable for any $\tau \geq 0$

(2) If equation (44) has a positive real root, when $\tau \in\left[0, \tau_{0}\right)$, the equilibrium $E^{*}$ is locally asymptotically stable, while when $\tau>\tau_{0}, E^{*}$ is unstable

(3) If $z_{k_{0}}$ is a single root of equation (44), then at $\tau=\tau_{0}$, system (1) will undergo a Hopf bifurcation

Proof

(1) If $G(z)=0$ has no positive real root, then the root of the characteristic equation on the left side of the imaginary axis is still there and does not cross the imaginary axis. Therefore, for any $\tau \geq 0$, the equilibrium $E^{*}$ is locally asymptotically stable.

(2) From the definition of $\tau_{0}$, it can be seen that there is no positive root of equation $G(z)=0$ in the set $\tau \in\left[0, \tau_{0}\right)$, and the root of its characteristic equation still does not cross the imaginary axis. Therefore, when $\tau \in\left[0, \tau_{0}\right)$, the equilibrium $E^{*}$ is locally asymptotically stable. When $\tau>\tau_{0}$, equation $G(z)=0$ has a root with a positive real part; thus, $E^{*}$ is unstable.

(3) If $z_{k_{0}}$ is a single root of equation $G(z)=0$, then $G^{\prime}\left(z_{k_{0}}\right) \neq 0 \quad$ and $\left.\quad(\operatorname{dRe}(\lambda) / \mathrm{d} \tau)\right|_{\tau=\tau_{0}} \neq 0 . \quad$ If $\left.(\mathrm{d} \operatorname{Re}(\lambda) / \mathrm{d} \tau)\right|_{\tau=\tau_{0}}<0$, then the root of the characteristic equation (38) at $\tau$ slightly less than $\tau_{0}$ has a positive real part, which contradicts the definition of $\tau_{0}$. Therefore, $\left.(\mathrm{d} \operatorname{Re}(\lambda) / \mathrm{d} \tau)\right|_{\tau=\tau_{0}}>0$ and $G^{\prime}\left(\omega_{0}^{2}\right)>0$. If $\left.(\mathrm{d} \operatorname{Re}(\lambda) / \mathrm{d} \tau)\right|_{\tau=\tau_{0}}>0$, when the value of $\tau$ is slightly less than $\tau_{0}$, it can be seen from Theorem 6 that all the roots of (38) have negative real parts. When the value of $\tau$ is slightly greater than $\tau_{0}$, equation (38) has the root with positive real part, which means that system (1) has Hopf bifurcation.

\section{Numerical Simulation}

In this section, the conclusions of the paper will be verified by numerical simulation and some of the conclusions will be extended. When selecting parameters, we refer to the parameter range in reference (see [24]), as shown in Table 1. 


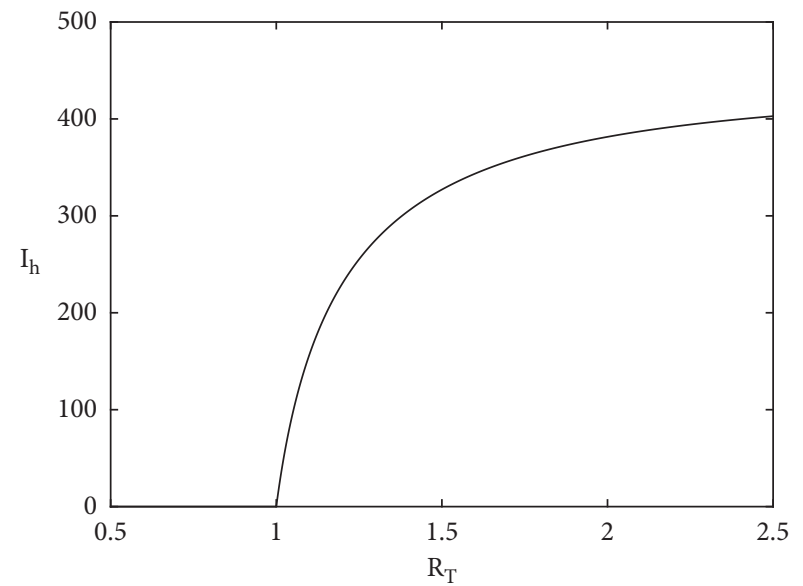

(a)

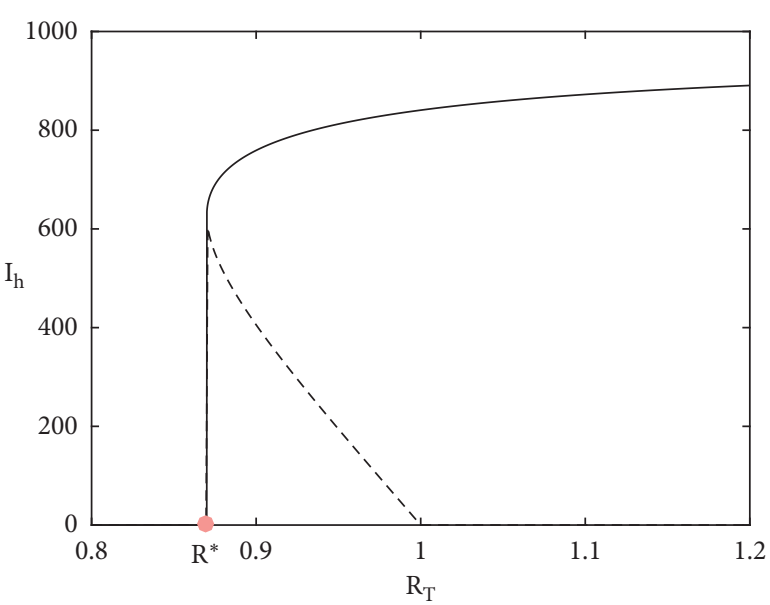

(b)

FIgURE 1: The bifurcation diagrams $(\mathrm{a}, \mathrm{b})$ show the change of the number of infected individuals $I_{h}$ with the control reproduction number $R_{T}$.

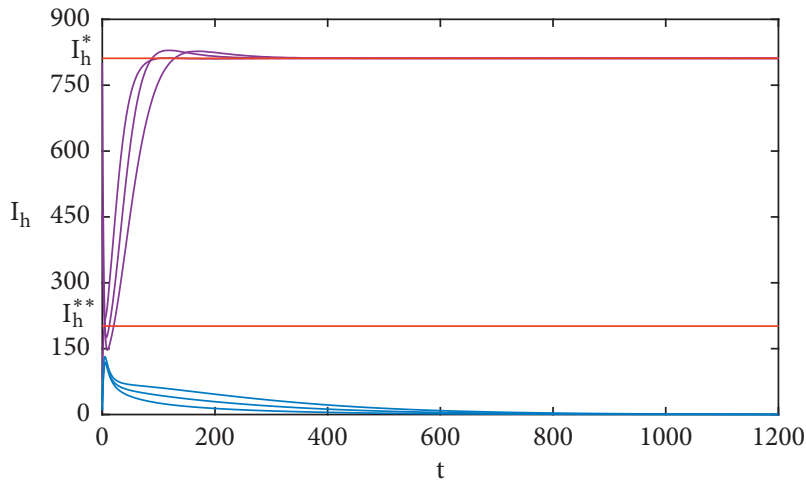

(a)

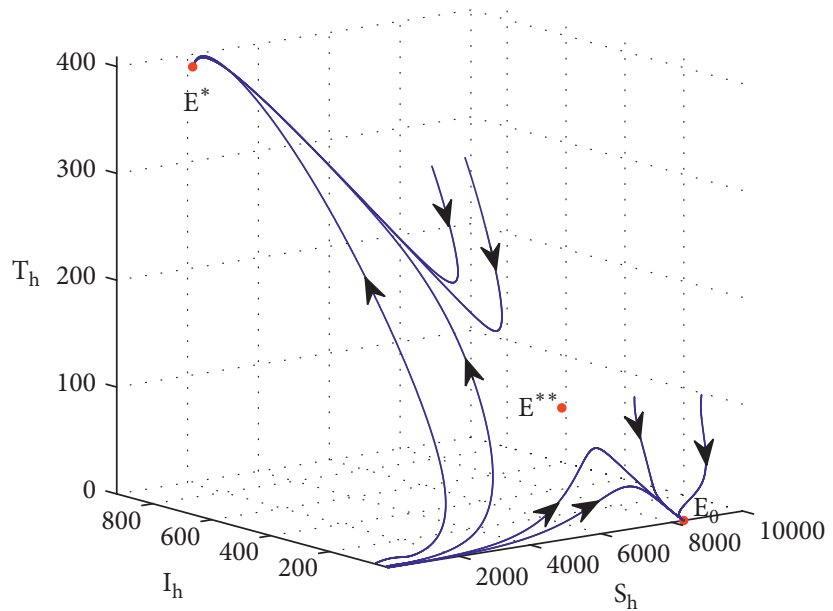

(b)

Figure 2: For different initial values, some trajectories tend to disease-free equilibrium $E_{0}$ and others to positive equilibrium $E^{*}$.

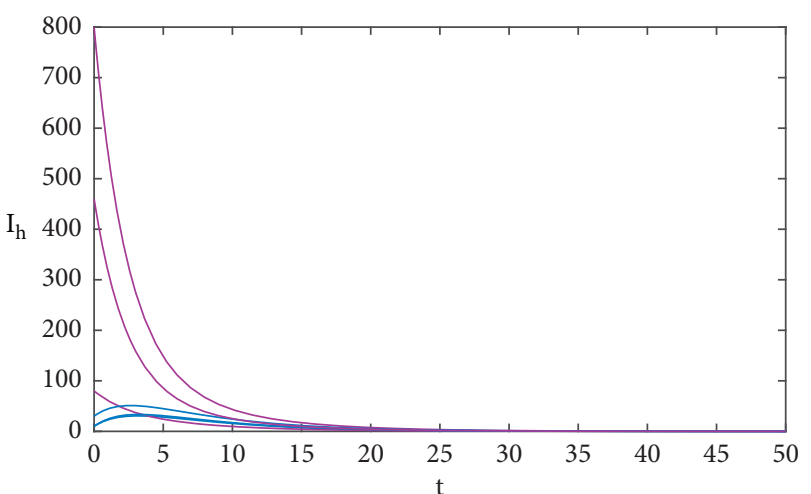

(a)

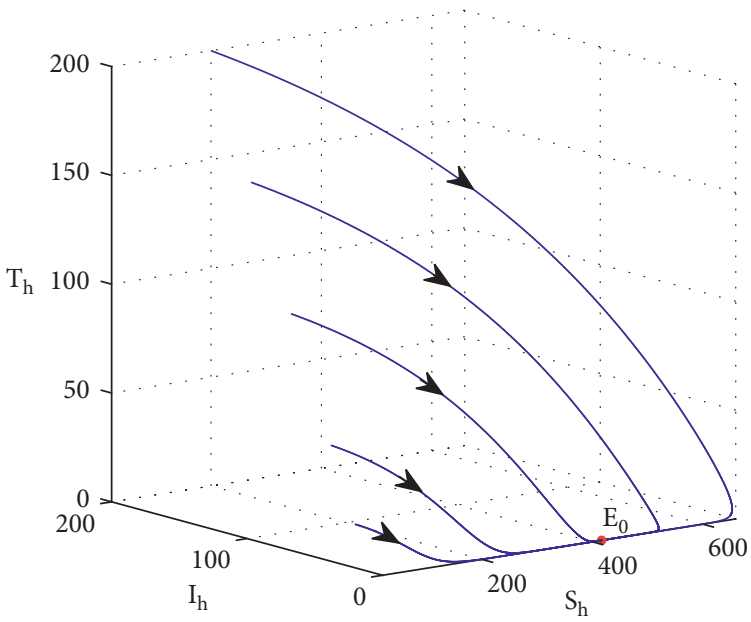

(b)

Figure 3: All trajectories tend to disease-free equilibrium $E_{0}$. 


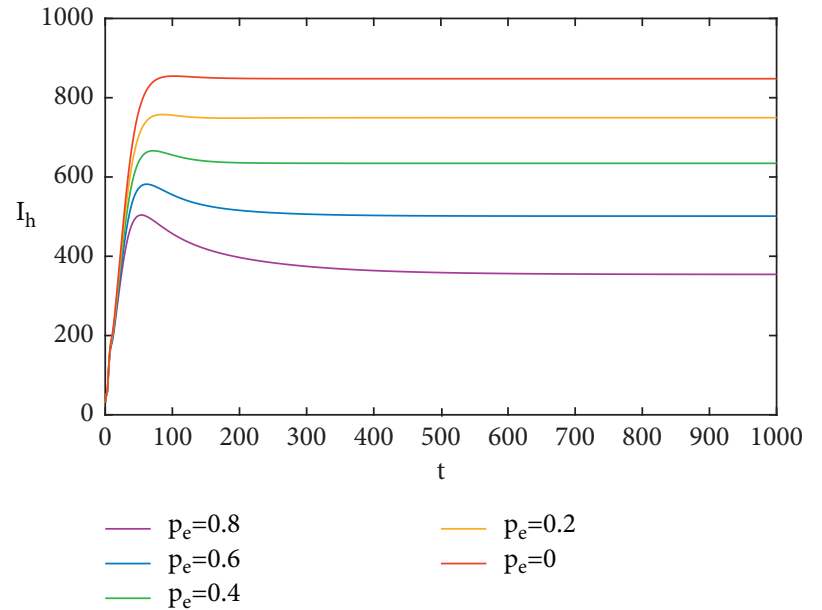

(a)

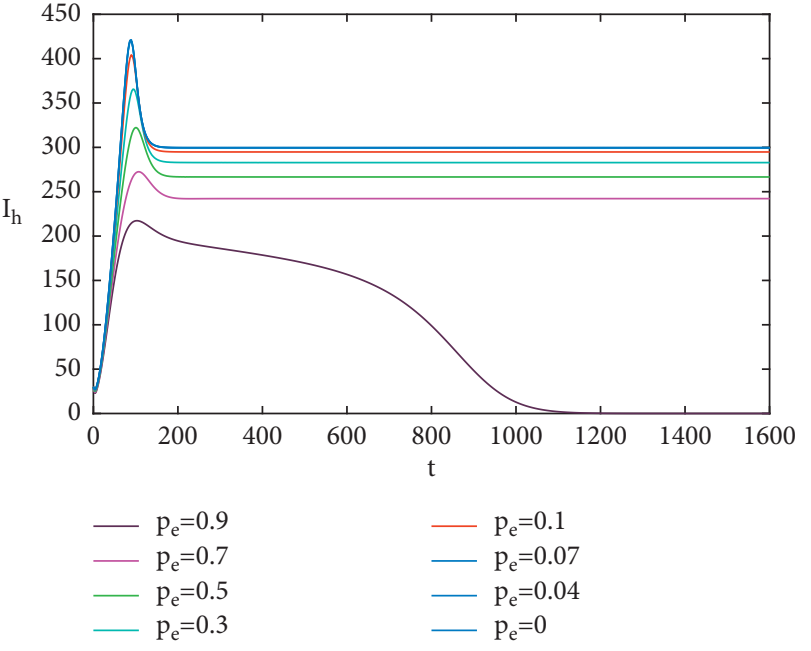

(b)

FIgURE 4: Effects of increased efficacy of TBDs $p_{e}$ on the number of malaria patients $I_{h}$.

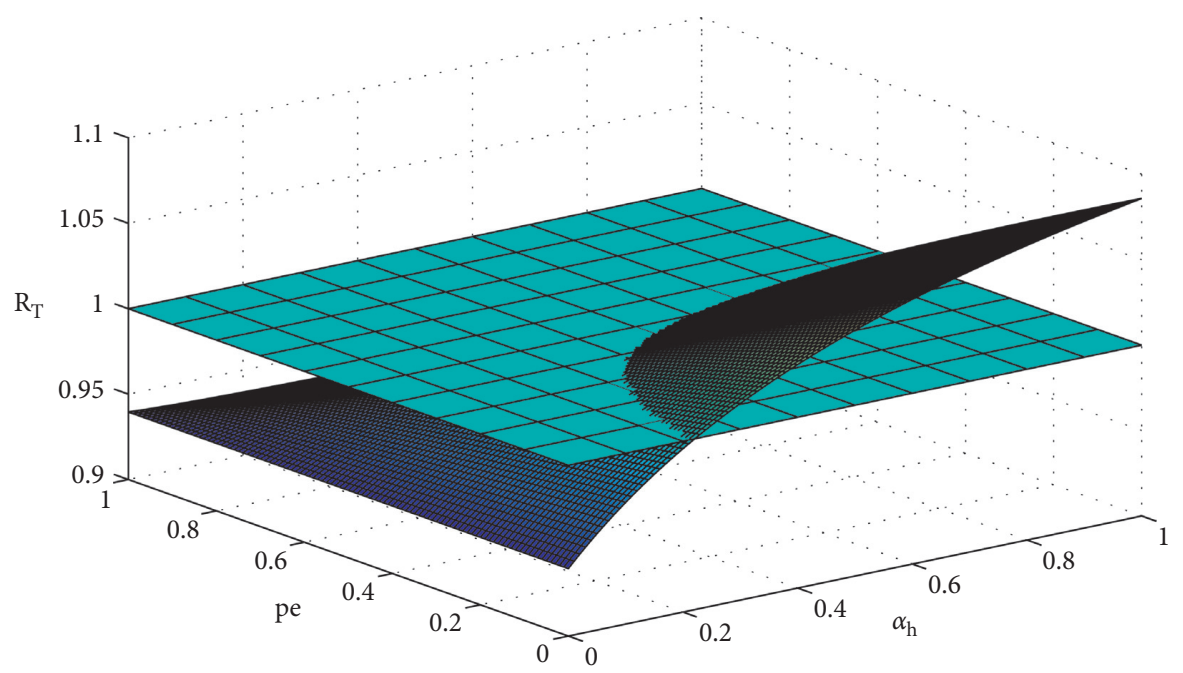

Figure 5: Diagram of the influence of $\alpha_{h}$ and $p_{e}$ on the control reproduction number $R_{T}$.

Let $\Lambda_{h}=100, d_{h}=0.012, d_{v}=0.08, \delta_{h}=0.08, \Lambda_{v}=200$, $r=5, \quad \rho_{h}=0.003, \quad \theta_{h}=0.2, \quad v=0.01, \quad \alpha_{h}=0.1, \quad \xi_{t}=0.4$, $\xi_{r}=0.2, \eta_{h}=0.1, \mu_{h}=0.2, p_{e}=0.1, \kappa_{h}=0.6$, and $\tau=3$. When $\gamma_{h}=0.01$, system (1) exhibits forward bifurcation (Figure 1(a)). When $\gamma_{h}=0.6$, system (1) exhibits backward bifurcation (Figure 1(b)); if $\rho_{v}=0.88$, then $R^{*}<R_{T}<1$; the system has a bistable phenomenon. The disease-free equilibrium $E_{0}$ and the positive equilibrium $E^{*}$ are locally asymptotically stable, while the positive equilibrium $E^{* *}$ is unstable (Figure 2).

Let $\Lambda_{h}=5, d_{h}=0.012, d_{v}=0.06, \delta_{h}=0.08, \gamma_{h}=0.6$, $\Lambda_{v}=100, r=0.1, \rho_{h}=0.002, \rho_{v}=0.005, \theta_{h}=0.2, v=0.01$, $\alpha_{h}=0.1, \quad \xi_{t}=0.4, \quad \xi_{r}=0.2, \quad \eta_{h}=0.1, \quad \mu_{h}=0.2, \quad p_{e}=0.1$, $\kappa_{h}=0.6$, and $\tau=0$; there is $R_{T}<\widetilde{R}<1$; the disease-free equilibrium $E_{0}$ is globally asymptotically stable (Figure 3 ). Compared with Figure 2, the selected initial values all tend to the disease-free equilibrium $E_{0}$.

In Figures $4(\mathrm{a})$ and $4(\mathrm{~b})$, the parameters $\gamma_{h}=0.9$, $\Lambda_{v}=200, r=5, \rho_{h}=0.003, \rho_{v}=0.9, \theta_{h}=0.2, v=0.01$, $\alpha_{h}=0.1, \xi_{t}=0.4, \xi_{r}=0.2, \eta_{h}=0.1, \mu_{h}=0.2$, and $\kappa_{h}=0.6$ are shared, and $p_{e}$ is a variable. In Figure $4(\mathrm{a})$, let $\Lambda_{h}=100$, $d_{h}=0.015, d_{v}=0.08, \delta_{h}=0.08$, and $\tau=3$. In Figure 4(b), let $\Lambda_{h}=80, d_{h}=0.013, d_{v}=0.09, \delta_{h}=0.24$, and $\tau=0.1$. When parameters meet conditions $E_{1}=-0.03202<0$, $E_{2}=-0.02078<0$, and $R_{T}>1$, according to Theorem 5 (1), with the increase of the efficacy of TBDs $p_{e} \in[0,1]$, the number of infected humans $I_{h}$ gradually decreased (Figure 4(a)). When parameters meet conditions $E_{1}=0.00133>0, E_{2}=-0.0182<0$, and $R^{*}<R_{T}<1$, take an initial value that tends to the equilibrium $E^{*}$. According to Theorem 5 (3), when $p_{e} \in[0,0.073]$, the improvement of the efficacy of TBDs had no significant effect on disease control, while when $p_{e} \in[0.073,1]$, the improvement of the efficacy of TBDs has a significant effect on disease control, and the trajectory eventually tends to the disease-free equilibrium $E_{0}$ (Figure 4(b)).

Let $d_{h}=0.013$, let $\alpha_{h}$ and $p_{e}$ be variables, and other parameters are the same as those in Figure 4(a). It can be 


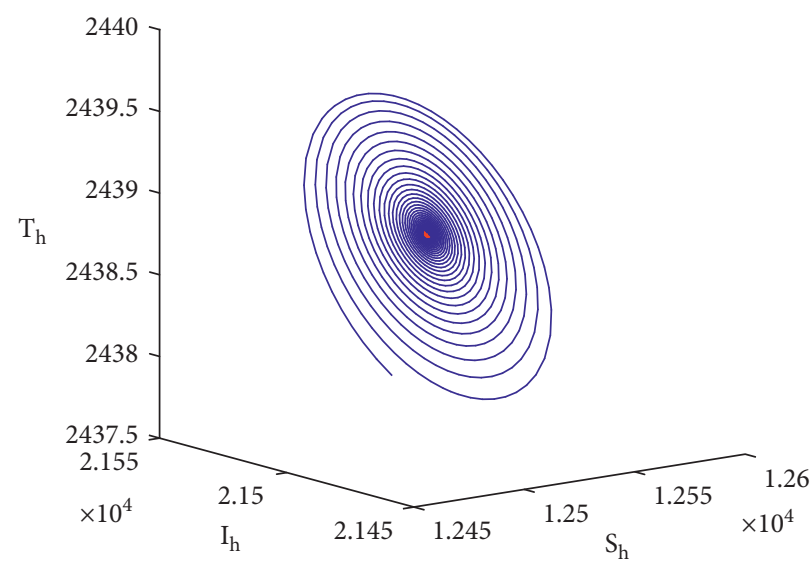

FIgURE 6: When $\tau=4.2$ is less than the threshold $\tau_{1}^{0}=4.48$, the positive equilibrium $E^{*}$ is locally asymptotically stable.

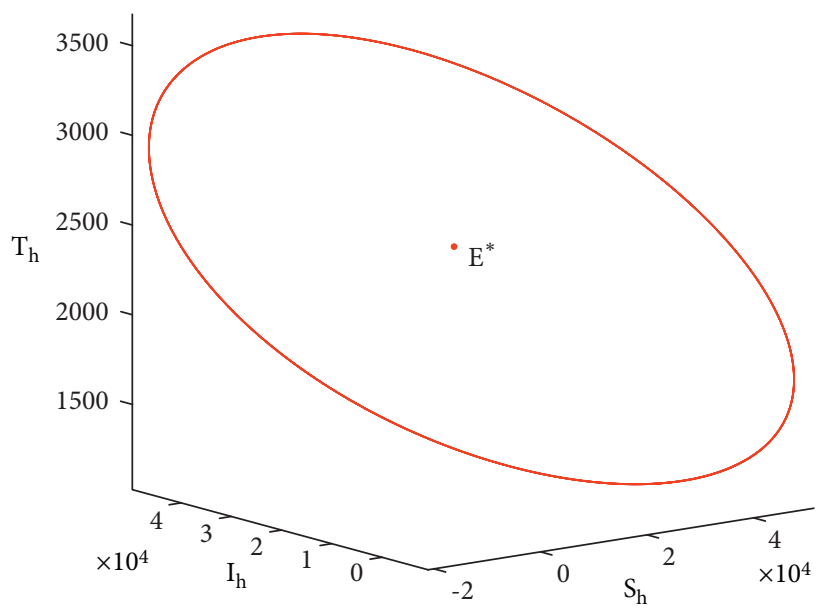

Figure 7: When $\tau=5$ is greater than the threshold $\tau_{1}^{0}=4.58, E^{*}$ is unstable and the system has a stable periodic solution.

found that when the treatment rate $\alpha_{h}$ is large enough and the efficacy of TBDs $p_{e}$ is low, the control reproduction number is still greater than 1 ; when $p_{e}$ is large enough, the control reproduction number is less than 1 (Figure 5).

Let $\Lambda_{h}=855, d_{h}=0.01, d_{v}=0.055, \delta_{h}=0.02, \gamma_{h}=0.8$, $\Lambda_{v}=2100, \quad r=5, \rho_{h}=0.003, \rho_{v}=0.8, \theta_{h}=0.2, v=0.2$, $\alpha_{h}=0.02, \xi_{t}=0.07, \xi_{r}=0.03, \eta_{h}=0.2, \mu_{h}=0.16, p_{e}=0.16$, and $\kappa_{h}=0.2$; there is $R_{T}>1$. In Figure 6, when $\tau=4.2$, there is only one positive real root in equation (44), and the bifurcation threshold $\tau_{1}^{0}=4.48$ is obtained. In this case, $\tau<\tau_{1}^{0}$, the positive equilibrium $E^{*}$ is locally asymptotically stable in Figure 6. In Figure 7, when $\tau=5$, only one positive real root exists in equation (44), and the bifurcation threshold is $\tau_{1}^{0}=4$.58. In this case, $\tau>\tau_{1}^{0}$, the positive equilibrium $E^{*}$ is unstable, and the system has a periodic solution. According to Theorem 8 , at $\tau_{1}^{0}$, system (1) undergoes a Hopf bifurcation.

\section{Conclusion}

In this paper, we establish a time-delay differential equation model with transmission-blocking drugs intervention, the effect of time delay on the stability of the equilibrium was obtained, and the effective range of treatment rate and the efficacy of TBDs under certain conditions was assessed. In the classification of equilibria, the model appears to have backward bifurcation. The bistable case suggests that disease may exist even if the control reproduction number is less than 1 , and the existence of the disease depends on the initial conditions. The global stability of the disease-free equilibrium provides a reference for malaria eradication under certain conditions. In addition, when the parameters meet some conditions, the treatment rate and the efficacy of TBDs play a positive role in disease control. In contrast, the increase in the efficacy of TBDs was more significant for disease control. It is found through Figure 4(b) that, in the bistable case, the trajectory that originally tends to the disease equilibrium starts to converge to the disease-free equilibrium after increasing the efficacy of TBDs $p_{e}$, that is, making the initial value of disease extinction larger in range with increasing the efficacy of TBDs $p_{e}$. Therefore, the development of the transmission-blocking drugs and the improvement of the efficacy of TBDs will play an important role in the long-term control of malaria. When considering the influence of time delay on the model, we find that when the control reproduction number is greater than 1 , the model exhibits Hopf bifurcation. When the time delay is less 
than the bifurcation value, the positive equilibrium is locally asymptotically stable; when the time delay is slightly greater than the bifurcation value, the positive equilibrium is unstable and the system has a periodic solution. The appearance of periodic solution corresponds to the phenomenon of malaria periodic outbreaks in reality.

\section{Appendix}

$$
\begin{aligned}
& r_{1}=\left.S_{h}^{*} \cdot \frac{\partial f_{h}}{\partial S_{h}}\right|_{E^{*}}=\left.S_{h}^{*} \cdot \frac{\partial f_{h}}{\partial I_{h}}\right|_{E^{*}}=\left.S_{h}^{*} \cdot \frac{\partial f_{h}}{\partial T_{h}}\right|_{E^{*}} \\
& =\left.S_{h}^{*} \cdot \frac{\partial f_{h}}{\partial P_{h}}\right|_{E^{*}}=\left.S_{h}^{*} \cdot \frac{\partial f_{h}}{\partial R_{h}}\right|_{E^{*}}=-f_{h}^{*} \frac{S_{h}^{*}}{N_{h}^{*}}, \\
& r_{2}=\left.S_{h}^{*} \cdot \frac{\partial f_{h}}{\partial I_{v}}\right|_{E^{*}}=f_{h}^{*} \frac{S_{h}^{*}}{I_{v}^{*}}, \\
& r_{3}=\left.S_{v}^{*} \cdot \frac{\partial f_{v}}{\partial S_{h}}\right|_{E^{*}}=\left.S_{v}^{*} \cdot \frac{\partial f_{v}}{\partial P_{h}}\right|_{E^{*}}=-f_{v}^{*} \cdot \frac{S_{v}^{*}}{N_{h}^{*}}, \\
& r_{4}=\left.S_{v}^{*} \cdot \frac{\partial f_{v}}{\partial I_{h}}\right|_{E^{*}}=\frac{S_{v}^{*}}{N_{h}^{*}} \\
& C_{1}=f_{h}^{*}+r_{1} \text {, } \\
& C_{2}=-v+r_{1} \text {, } \\
& C_{3}=-\gamma_{h}+r_{1} \text {, } \\
& C_{4}=-\kappa_{h} \psi_{2} \text {, } \\
& C_{5}=-\left(1-\kappa_{h}\right) \psi_{2} \text {, } \\
& C_{6}=r_{3}+r_{4} \text {, } \\
& C_{7}=r_{3}+\xi_{t} r_{4} \text {, } \\
& C_{8}=r_{3}+\xi_{r} r_{4} \text {, } \\
& C_{9}=f_{v}^{*}+d_{v} \\
& D_{11}=\alpha_{h} C_{1} \text {, } \\
& D_{12}=d_{v}+C_{9} \text {, } \\
& D_{13}=d_{v} C_{9} \text {, } \\
& D_{14}=C_{4}\left(T_{2}+T_{3}\right)+\gamma_{h} C_{5}-v \psi_{1} \text {, } \\
& D_{15}=C_{4} T_{2} T_{3}+\gamma_{h} C_{5} T_{2}-v \psi_{1} T_{3} \text {, } \\
& D_{21}=-\alpha_{h} \gamma_{1} \text {, } \\
& D_{22}=d_{v}+C_{9}+d_{h} \text {, } \\
& D_{23}=d_{v} C_{9}+d_{v} d_{h}+C_{9} d_{h} \text {, } \\
& D_{24}=d_{v} C_{9} d_{h},
\end{aligned}
$$

$$
\begin{aligned}
& D_{25}=T_{2}+T_{3}-C_{5}+\psi_{1} \text {, } \\
& D_{26}=T_{2} T_{3}-T_{2} C_{5}+\psi_{1} T_{3} \text {, } \\
& D_{31}=d_{v}+T_{1}+T_{2}+C_{9} \text {, } \\
& D_{32}=d_{v}\left(T_{1}+T_{2}+C_{9}\right)+C_{9}\left(T_{1}+T_{2}\right)+T_{1} T_{2} \text {, } \\
& D_{33}=d_{v} C_{9} T_{1}+d_{v} C_{9} T_{2}+d_{v} T_{1} T_{2}+C_{9} T_{1} T_{2} \text {, } \\
& D_{34}=d_{v} T_{1} T_{2} C_{9} \text {, } \\
& D_{35}=C_{1}-r_{1} \text {, } \\
& D_{36}=C_{1}\left(\beta_{1 h}+T_{3}\right)-\theta_{h} r_{1}-r_{1}\left(d_{h}+T_{3}\right), \\
& D_{37}=C_{1} \beta_{1 h} T_{3}-\theta_{h} r_{1} d_{h}-r_{1} d_{h} T_{3}-C_{1} \theta_{h} \gamma_{h} \text {, } \\
& D_{41}=-r_{2} \psi_{1} \alpha_{h} \text {, } \\
& D_{42}=d_{v}+T_{3} \text {, } \\
& D_{43}=d_{v} T_{3} \text {, } \\
& D_{44}=r_{3}\left(d_{h}+v\right) \text {, } \\
& D_{51}=r_{2} \alpha_{h} \text {, } \\
& D_{52}=d_{v}+T_{2} \text {, } \\
& D_{53}=d_{v} T_{2} \text {, } \\
& D_{54}=-C_{7} \text {, } \\
& D_{55}=-C_{7}\left(d_{h}+T_{3}\right)+C_{5} C_{8}+r_{3} r_{4} \text {, } \\
& D_{56}=-C_{7} d_{h} T_{3}+C_{5} C_{8} d_{h}+r_{3} C_{4} T_{3}+r_{3} C_{5} \gamma_{h} \text {, } \\
& D_{61}=r_{2} \text {, } \\
& D_{62}=d_{v}+T_{1}+T_{2} \text {, } \\
& D_{63}=d_{v} T_{2}+d_{v} T_{1}+T_{1} T_{2} \text {, } \\
& D_{64}=d_{v} T_{1} T_{2} \text {, } \\
& D_{65}=r_{3}-C_{6} \text {, } \\
& D_{66}=r_{3}\left(\beta_{1 h}+T_{3}\right)-C_{6}\left(d_{h}+T_{3}\right)-\theta_{h} C_{8} \text {, } \\
& D_{67}=-\theta_{h} C_{8} d_{h}+r_{3} \beta_{1 h} T_{3}-r_{3} \theta_{h} \gamma_{h}-C_{6} d_{h} T_{3} \text {, } \\
& D_{71}=d_{v}+C_{9}+d_{h} \text {, } \\
& D_{72}=d_{v} C_{9}+C_{9} d_{h}+d_{v} d_{h} \text {, } \\
& D_{73}=d_{v} C_{9} d_{h} \text {, } \\
& D_{74}=T_{2}+T_{3} \text {, } \\
& D_{75}=T_{2} T_{3} \text {, } \\
& D_{76}=\beta_{1 h}+T_{1} \text {, } \\
& D_{77}=T_{1} \beta_{1 h}+\alpha_{h} C_{4} \text {, } \\
& F_{6}=D_{76}+D_{74}+D_{71} \text {, } \\
& F_{5}=D_{77}+D_{76}\left(D_{74}+D_{71}\right)+D_{75}+D_{72}+D_{71} D_{74} \text {, } \\
& F_{4}=D_{77}\left(D_{74}+D_{71}\right)+D_{76}\left(D_{75}+D_{72}+D_{71} D_{74}\right) \\
& +D_{71} D_{75}+D_{72} D_{74}+D_{73} \text {, } \\
& F_{3}=D_{77}\left(D_{75}+D_{72}+D_{71} D_{74}\right) \\
& +D_{76}\left(D_{71} D_{75}+D_{72} D_{74}+D_{73}\right)+\left(D_{72} D_{75}+D_{73} D_{74}\right) \text {, } \\
& F_{2}=D_{77}\left(D_{71} D_{75}+D_{72} D_{74}+D_{73}\right) \\
& +D_{76}\left(D_{72} D_{75}+D_{73} D_{74}\right)+D_{73} D_{75} \text {, } \\
& F_{1}=D_{77}\left(D_{72} D_{75}+D_{73} D_{74}\right)+D_{76} D_{73} D_{75} \text {, } \\
& F_{0}=D_{73} D_{75} D_{77} \\
& J_{6}=D_{35} \text {, } \\
& J_{5}=D_{21}+D_{36}+D_{31} D_{35}+D_{61} D_{65},
\end{aligned}
$$




$$
\begin{aligned}
J_{4}= & D_{11} C_{4}+D_{21}\left(D_{25}+D_{22}\right)+D_{37}+D_{31} D_{36}+D_{32} D_{35} \\
& +D_{51} D_{54}+D_{61}\left(D_{66}+D_{62} D_{65}\right), \\
J_{3}= & D_{11}\left(D_{14}+D_{12} C_{4}\right)+D_{21}\left(D_{26}+D_{22} D_{25}+D_{23}\right) \\
& +D_{31} D_{37}+D_{32} D_{36}+D_{33} D_{35} \\
& +D_{41} r_{3}+D_{51}\left(D_{55}+D_{52} D_{54}\right) \\
& +D_{61}\left(D_{67}+D_{62} D_{66}+D_{63} D_{65}\right), \\
J_{2}= & D_{11}\left(D_{15}+D_{12} D_{14}+D_{13} C_{4}\right) \\
& +D_{21}\left(D_{24}+D_{22} D_{26}+D_{23} D_{25}\right) \\
& +D_{32} D_{37}+D_{33} D_{36}+D_{34} D_{35}+D_{41}\left(D_{44}+D_{42} r_{3}\right) \\
& +D_{51}\left(D_{56}+D_{52} D_{55}+D_{53} D_{54}\right) \\
& +D_{61}\left(D_{62} D_{67}+D_{63} D_{66}+D_{64} D_{65}\right), \\
J_{1}= & D_{11}\left(D_{12} D_{15}+D_{13} D_{14}\right)+D_{21}\left(D_{23} D_{26}+D_{24} D_{25}\right) \\
& +D_{33} D_{37}+D_{34} D_{36}+D_{41}\left(D_{42} D_{44}+D_{43} r_{3}\right) \\
& +D_{51}\left(D_{52} D_{56}+D_{53} D_{55}\right)+D_{61}\left(D_{63} D_{67}+D_{64} D_{66}\right), \\
J_{0}= & D_{11} D_{13} D_{15}+D_{21} D_{24} D_{26}+D_{34} D_{37}+D_{41} D_{43} D_{44} \\
& +D_{51} D_{53} D_{56}+D_{61} D_{64} D_{67} .
\end{aligned}
$$

\section{Data Availability}

Previously reported data were used to support this study. These prior datasets are cited at relevant places within the text as references.

\section{Conflicts of Interest}

The authors declare that they have no conflicts of interest.

\section{Acknowledgments}

This work was supported by the National Natural Science Foundation of China (11971055).

\section{References}

[1] A. M. Noor, D. K. Kinyoki, C. W. Mundia et al., "The changing risk of Plasmodium falciparum malaria infection in Africa: 2000-10: a spatial and temporal analysis of transmission intensity," The Lancet, vol. 383, no. 9930, pp. 1739-1747, 2014.

[2] C. J. Murray, L. C. Rosenfeld, S. S. Lim et al., "Global malaria mortality between 1980 and 2010: a systematic analysis," The Lancet, vol. 379, no. 9814, pp. 413-431, 2012.

[3] J. Landier, D. M. Parker, A. M. Thu et al., "Effect of generalised access to early diagnosis and treatment and targeted mass drug administration on Plasmodium falciparum malaria in Eastern Myanmar: an observational study of a regional elimination programme," The Lancet, vol. 391, no. 10133, pp. 1916-1926, 2018.

[4] M. Imwong, T. T. Hien, N. T. Thuy-Nhien, A. M. Dondorp, and N. J. White, "Spread of a single multidrug resistant malaria parasite lineage (PfPailin) to Vietnam," The Lancet Infectious Diseases, vol. 17, no. 10, pp. 1022-1023, 2017.

[5] R. W. Van Der Pluijm, M. Imwong, N. H. Chau et al., "Determinants of dihydroartemisinin-piperaquine treatment failure in Plasmodium falciparum malaria in Cambodia,
Thailand, and Vietnam: a prospective clinical, pharmacological, and genetic study," The Lancet Infectious Diseases, vol. 19, no. 9, pp. 952-961, 2019.

[6] L.-M. Birkholtz, T. L. Coetzer, D. Mancama, D. Leroy, and P. Alano, "Discovering new transmission-blocking antimalarial compounds: challenges and opportunities," Trends in Parasitology, vol. 32, no. 9, pp. 669-681, 2016.

[7] M. J. Delves, F. Angrisano, and A. M. Blagborough, "Antimalarial transmission-blocking interventions: past, present, and future," Trends in Parasitology, vol. 34, no. 9, pp. 735-746, 2018.

[8] D. M. Plouffe, M. Wree, AY. Du, S. Meister, and F. Li, K. Patra, A. Lubar, S. L. Okitsu et al., High-throughput assay and discovery of small molecules that interrupt malaria transmission," Cell Host and Microbe, vol. 19, pp. 114-126, 2016.

[9] R. Ross, The Prevention of Malaria, John Murray, London, UK, 1911.

[10] G. Macdonald, "The analysis of infection rates in diseases in which superinfection occurs," Tropical Diseases Bulletin, vol. 47, pp. 907-915, 1950.

[11] J. L. Aron, "Mathematical modelling of immunity to malaria," Mathematical Biosciences, vol. 90, no. 1-2, pp. 385-396, 1988.

[12] P. Auger, E. Kouokam, G. Sallet, M. Tchuente, and B. Tsanou, "The Ross-MacDonald model in a patchy environment," Mathematical Biosciences, vol. 216, no. 2, pp. 123-131, 2008.

[13] D. Gao and S. Ruan, "A multipatch malaria model with logistic growth populations," SIAM Journal on Applied Mathematics, vol. 72, no. 3, pp. 819-841, 2012.

[14] Y. Lou and X.-Q. Zhao, "A reaction-diffusion malaria model with incubation period in the vector population," Journal of Mathematical Biology, vol. 62, no. 4, pp. 543-568, 2011.

[15] G. Macdonald, The Epidemiology and Control of Malaria, Oxford University Press, Oxford, UK, 1957.

[16] G. A. Ngwa, "Modelling the dynamics of endemic malaria in growing populations," Discrete and Continuous Dynamical Systems - B, vol. 4, no. 4, pp. 1173-1202, 2004.

[17] G. A. Ngwa and W. S. Shu, "A mathematical model for endemic malaria with variable human and mosquito populations," Mathematical and Computer Modelling, vol. 32, no. 7-8, pp. 747-763, 2000.

[18] N. Chitnis, J. M. Cushing, and J. M. Hyman, "Bifurcation analysis of a mathematical model for malaria transmission," SIAM Journal on Applied Mathematics, vol. 67, no. 1, pp. 24-45, 2006.

[19] N. Chitnis, J. M. Hyman, and J. M. Cushing, "Determining important parameters in the spread of malaria through the sensitivity analysis of a mathematical model," Bulletin of Mathematical Biology, vol. 70, no. 5, pp. 1272-1296, 2008.

[20] S. Ruan, D. Xiao, and J. C. Beier, "On the delayed RossMacDonald model for malaria transmission," Bulletin of Mathematical Biology, vol. 70, no. 4, pp. 1098-1114, 2008.

[21] R. Zhao and J. Mohammed-Awel, "A mathematical model studying mosquito-stage transmission-blocking vaccines," Mathematical Biosciences and Engineering, vol. 11, no. 5, pp. 1229-1245, 2014.

[22] M. I. Teboh-Ewungkem, C. N. Podder, and A. B. Gumel, "Mathematical study of the role of gametocytes and an imperfect vaccine on malaria transmission dynamics," Bulletin of Mathematical Biology, vol. 72, no. 1, pp. 63-93, 2010.

[23] H. C. Slater, L. C. Okell, and A. C. Ghani, "Mathematical modelling to guide drug development for malaria elimination," Trends in Parasitology, vol. 33, no. 3, pp. 175-184, 2017.

[24] W. A. Woldegerima, R. Ouifki, and J. Banasiak, "Mathematical analysis of the impact of transmission-blocking drugs 
on the population dynamics of malaria," Applied Mathematics and Computation, vol. 400, Article ID 126005, 2021.

[25] C. Chiyaka, Z. Mukandavire, and P. Das, "Global dynamics of a malaria model with partial immunity and two discrete time delays," International Journal of Biomathematics, vol. 4, no. 2, pp. 135-147, 2011.

[26] H. Wan and J.-A. Cui, "A malaria model with two delays," Discrete Dynamics in Nature and Society, vol. 2013, Article ID 601265, 8 pages, 2013. 